\title{
Angiography for Peripheral Vascular Intervention
}

\author{
Yoshiaki Yokoi \\ Cardiology, Kishiwada Tokushuaki Hospital, Osaka \\ Japan
}

\section{Introduction}

The use of endovascular technique for peripheral vascular disease (PVD) has evolved and new devices and techniques were being developed. Even after recent development of noninvasive diagnosis of PVD, angiography is still the gold standard for the evaluation of stenotic and occluded lesions. Without precise angiographic information, endovascular therapy can not be performed. In this chapter, the angiographic technique to visualize major arteries of atherosclerotic disease is described.

\section{Angiography suite for peripheral vascular intervention}

Excellent imaging is the key for the success of endovascular therapies. Flat-panel $\mathrm{X}$ ray image detectors for use in digital fluoroscopy and angiography are essential for peripheral artery intervention. The ability of three-dimensional (3D) visualization techniques and bolus chasing are also required. Various types of imaging sizes are available but we have to compromise considering of function on the machine. Careful planning and professional expertise is a key factor for choosing every endovascular suite.

\subsection{Single plane vs. biplane system}

We have two types of our peripheral angiography suite. One is single plane arm with $31 \mathrm{~cm}$ $X 31 \mathrm{~cm}$ flat panel detector (Fig. Suite1A). And the other biplane with the $30 \mathrm{~cm} \mathrm{X} 40 \mathrm{~cm}$ flat panel detector (Fig. Suite1B). Both machines have its merit and demerit. Single plane machine has the versatile function and more suited for intervention. Biplane system could obtain the multiple images and reduce the dosage of dye. But except for cerebral angiography, it takes time to adjust two images in centred position. For the diagnostic purpose, biplane System has the advantage, but interventional work, the single plane system has more versatile function and much safer for the patient's care during procedure.

\subsection{Extra monitor}

In angiographic table, operators usually stand on the right side, but for left limb intervention or left brachial approach, operator needs to be positioned on the left side of the table. In these circumstances, the extra monitor is useful for left side operator. Without moving central image monitor, main operator can do the procedure with assistant who are seeing central monitor from right side (Fig. Suite2A). This is the convenient way to intervene 
right femoropopliteal artery or left subclavian artery. In left below the knee procedure via cross over approach, C-arm is rotated to the left side. Cranial side operator may not see the central image. In this situation, extra monitor can be placed in left cranial side (Fig. Suite2B).
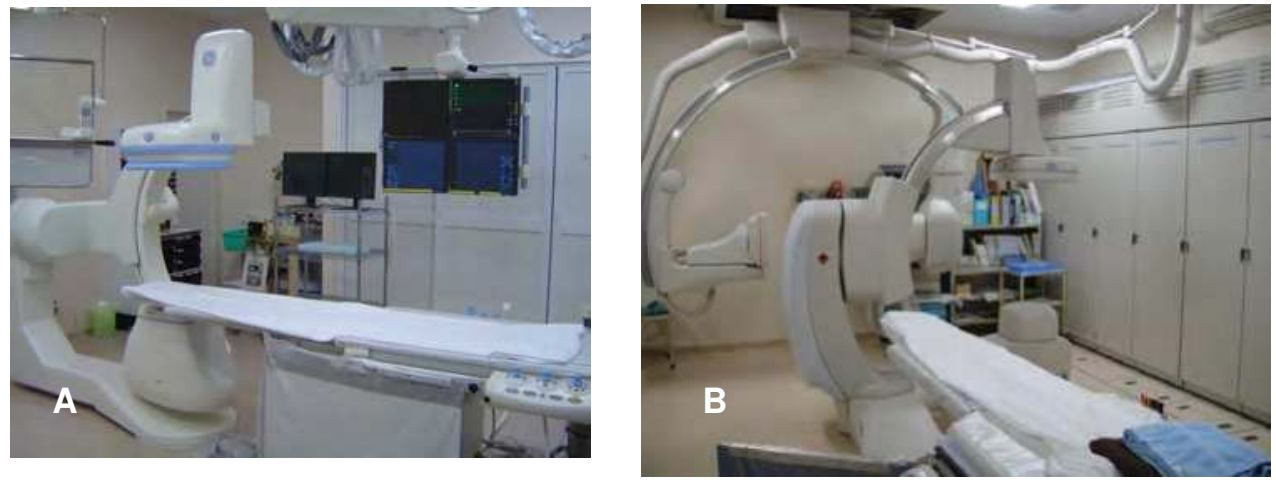

Fig. Angiosuite 1: Peripheral angiography suite

A: Single plane flat panel system.

A single plane system has more versatile function compared to the biplane system. Single system is suited for interventional work

B: Biplane system: Biplane system allows the two images both on fluoro and images. Dye consumption could reduce. However, lateral tube might limit the patient's care. Mostly employed for neuroangiography.
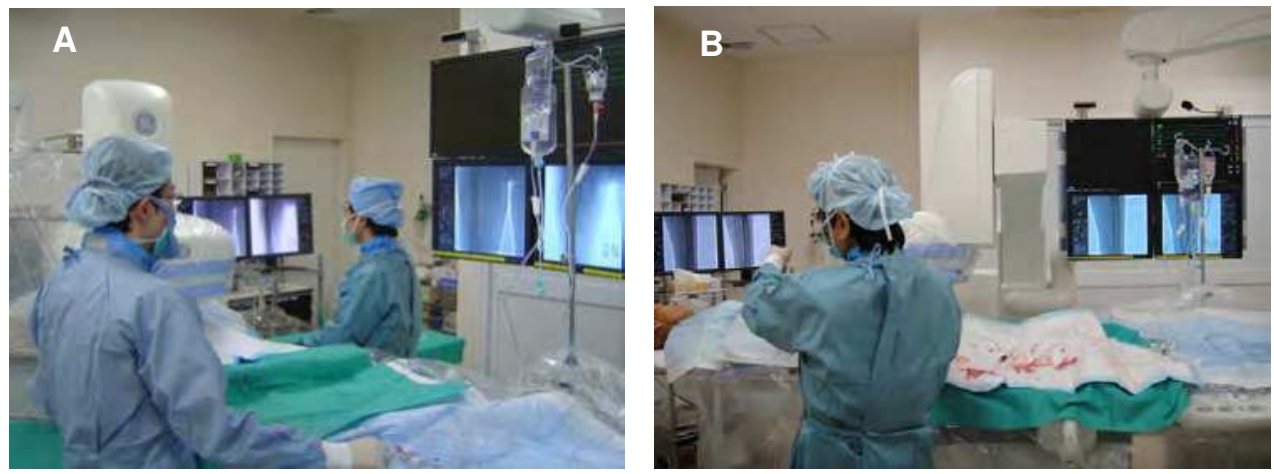

Fig. Angiosuite 2: Extra monitor

A: Operators usually stand on the right side of the table. But for left limb intervention or left brachial approach, operator needs to be positioned on the left side of the table. In these circumstances, the extra monitor is useful for left side standing operator without moving central image monitor

B: In left below the knee procedure via cross over approach, $\mathrm{C}$-arm is rotated to the left side. Cranial side operator may not see the central image. In this situation, extra monitor can be placed in left cranial side. Primary operator could see the image on the cranial side monitor and assistant could see the central monitor. 


\subsection{Power injectors}

For most of small vessel and selective angiography, hand injection is adequate. However, for the optimal opacification of high-flow blood vessels like aorta, the use of power injector is mandatory. Constant and high volume of dye should be injected through electronically calibrated power injector. There are two types of injector. One is old fashioned power injector and the other is the assisted device which could give the small or large amount of dye by the injector attached to catheter table. Any contrast volume is adjusted by manually. Even a small dose of dye can be injected. However, the space of left side of table is occupied by this assisted device. We prefer conventional power injector which is mounted to the celling. This method gives us more space around catheter table.

In the assisted device, the operator is supposed to stand only on the right side of the table and can not be away from the table during injection. To prevent for radiation exposure, conventional use of power injector is more preferable (Fig. Suite4).

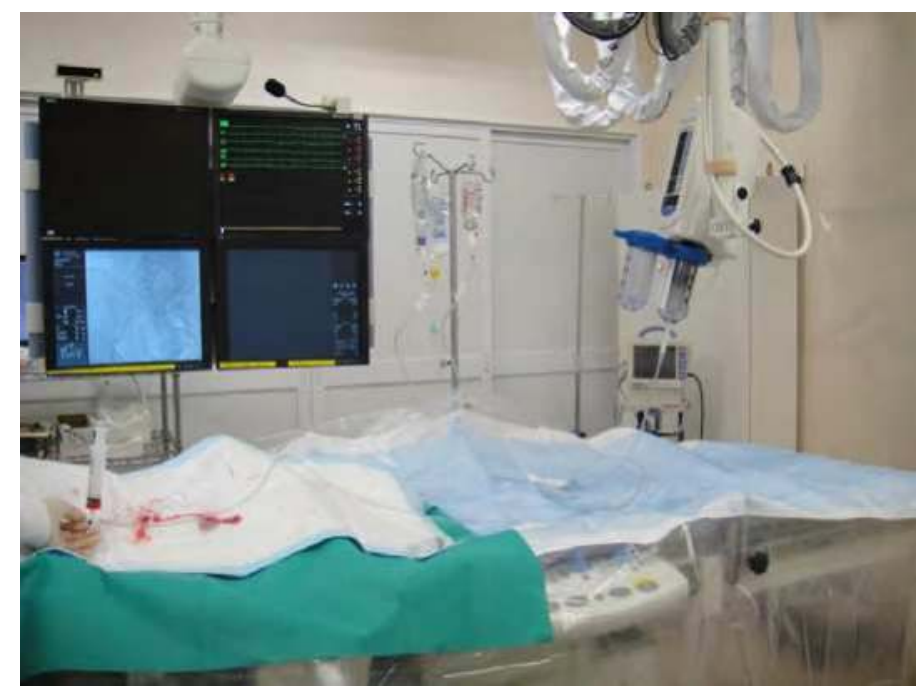

Fig. Angiosuite 3: Celling mounted Power injector

Celling mounted power injector gives more space around catheter table

\subsection{Image size and contrast volume}

Contrast volume for opacification of the major arteries were listed in Table 1.was listed. These injection volumes are mainly used in our catheter laboratory. But, real contrast volume depends on the patient' condition, catheter size, amount of contrast, speed of injection and et al. Therefore contrast dose should be individurized on each case 


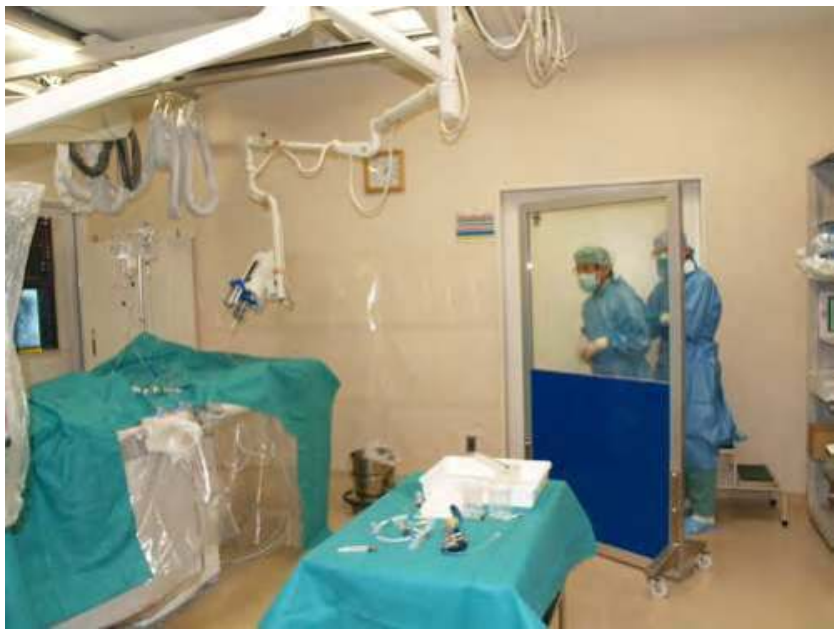

Fig. Angiosuite4: Radiation exposure

Radiation exposure should be minimalized during injection of dye by using power injector

\subsection{Table 1 Commonly used imaging size, injection rates and contrast volume for major arteries}

\begin{tabular}{|l|c|c|c|}
\hline Angiogram & Image size & Injection rate & $\begin{array}{c}\text { Contrast } \\
\text { volume }\end{array}$ \\
\hline Aortic Arch & $30 \mathrm{~cm}$ & $20 \mathrm{cc} / \mathrm{s}$ & $20-30 \mathrm{cc}$ \\
\hline Carotid (Selective) & $20 \mathrm{~cm}$ & $4-6 \mathrm{cc} / \mathrm{s}$ & $7-9 \mathrm{cc}$ \\
\hline Cerebral: AP & $20 \mathrm{~cm}$ & $4-6 \mathrm{cc} / \mathrm{s}$ & $7-9 \mathrm{cc}$ \\
\hline Cerebral: Lateral & $30 \mathrm{~cm}$ & $4-6 \mathrm{cc} / \mathrm{s}$ & $7-9 \mathrm{cc}$ \\
\hline Abdominal aorta & $20-40 \mathrm{~cm}$ & $16-20 \mathrm{cc} / \mathrm{s}$ & $16-25 \mathrm{cc}$ \\
\hline Renal (Selective) & $20 \mathrm{~cm}$ & $4-6 \mathrm{cc} / \mathrm{s}(\mathrm{by}$ hand) & $4-6 \mathrm{cc}$ \\
\hline Iliac (Selective) & $20 \mathrm{~cm}$ & $6-10 \mathrm{cc} / \mathrm{s}$ & $8-10 \mathrm{cc}$ \\
\hline Femoropopliteal-Tibial (Bolus) 30cm & $4 \mathrm{cc} / \mathrm{s}$ & $16-20$ & \\
\hline SFA & $30 \mathrm{~cm}$ & $4-6 \mathrm{cc} / \mathrm{s}$ & $10 \mathrm{cc}$ \\
\hline SFA (Selective) & $20-30 \mathrm{~cm}$ & $4-6 \mathrm{cc} / \mathrm{s}$ & $10 \mathrm{cc}$ \\
\hline Below the knee (Bolus) & $30 \mathrm{~cm}$ & $3 \mathrm{cc} / \mathrm{s}$ & $7-9 \mathrm{cc}$ \\
\hline Tibial & $20 \mathrm{~cm}$ & $3 \mathrm{cc} / \mathrm{s}$ & $5 \mathrm{cc}$ \\
\hline Tibial (Selective) & $20 \mathrm{~cm}$ & $1-2 \mathrm{cc} / \mathrm{s}(\mathrm{by}$ hand) & $2-3 \mathrm{cc}$ \\
\hline
\end{tabular}

Table 1. Commonly used imaging size, injection rates and contrast volume AP: antero-posterior, SFA: superficial femoral artery

\section{Carotid artery angiography}

The atherosclerotic plaque accumulates at the carotid bifurcation. There are a number of factors, including geometry, velocity profile, and shear stress. The carotid bulb forms a focal dilatation. The flow at the bifurcation is considered to be a complex hemodynamics and postulated to lead carotid artery stenosis (Fig. Carotid 1) (1). Carotid disease is one of 
important cause of ischemic stroke. In symptomatic patients, carotid revascularization is indicated in the presence of a stenosis 50 70\% or more (2). There are many arguments about the indication for asymptomatic patients. But asymptomatic stenoses are usually treated only if luminal narrowing exceeds 60\%(2). Carotid artery stenting (CAS) is a preferred treatment strategy in high-risk patients requiring Carotid endoatherectomy (CEA). Even though indication of CAS is still controversial, CAS is a less invasive and attractive way for revascularization for carotid artery disease. To indicate carotid artery revascularization, meticulous angiographic approach should be taken to evaluate of carotid artery stenosis.
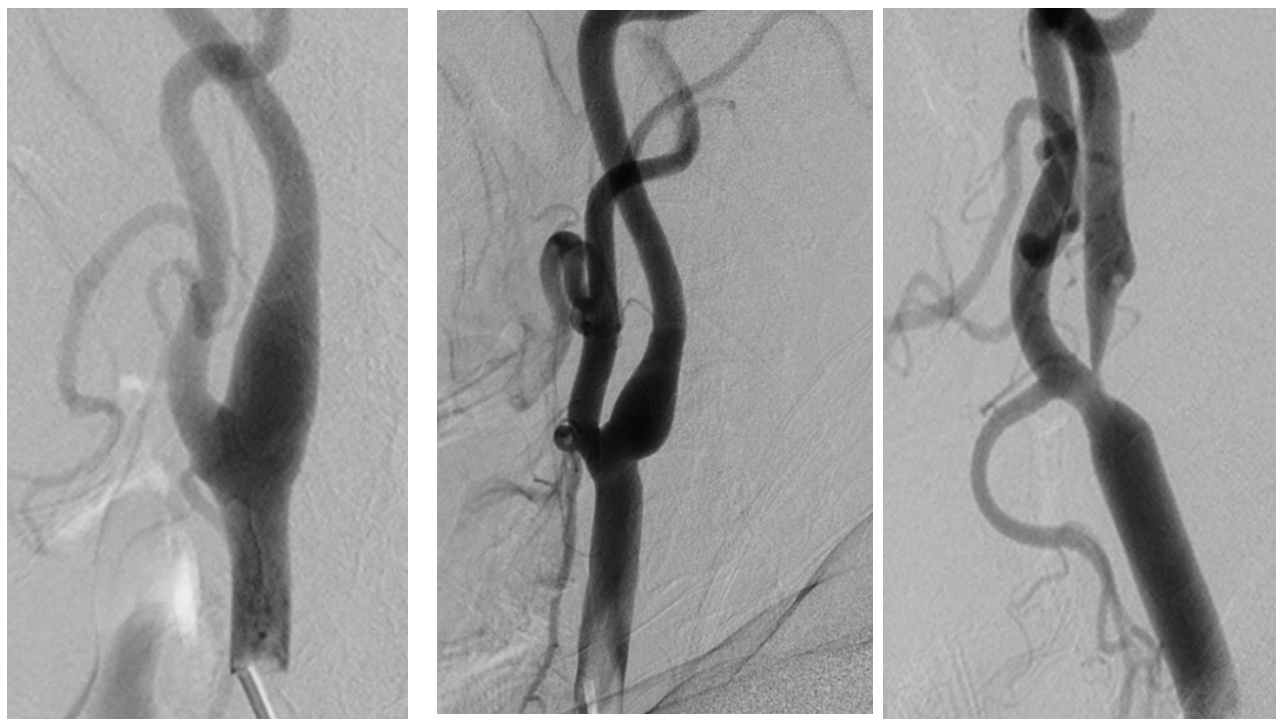

Fig. Carotid 1: Progression of carotid artery disease

A: The carotid bulb forms a focal dilatation

B: Mostly lesion starts at the bifurcation. Mild stenosis is seen

C: In progressive form of atherosclerosis.

Carotid bulb is filled with plaque and the flow at the bifurcation show the complex

hemodynamics. This hemodynamics is postulated to lead carotid artery stenosis

\subsection{Aortic arch type}

Engagement of catheter to the brachiocephalic or left CCA is required to perform carotid artery angiography. In selecting catheter, the aortic arch anatomy plays an important role for the success of the procedure. In CAS, not only engaging the catheter, guiding sheath have to be placed into common CCA. It does mean the technique of the deep engagement of diagnostic catheter to CCA or external carotid artery (ECA) is the important procedure for carotid artery angiography.

There are classifications that categorize aortic arch anatomy (3) (Fig. Carotid 2).

The aortic arch angiography in left anterior oblique view shows three type of aortic arch (Fig. Carotid 3). The vertical distance from the origin of the innominate artery to the top of the arch determines the arch type. In type 3, engagement of catheter, particularly to left common carotid artery is difficult and may account for the failure of carotid artery stenting. 


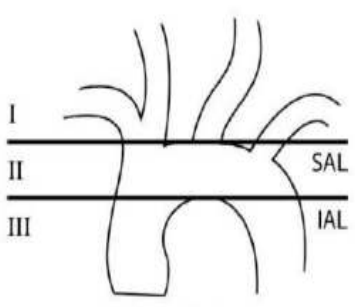

Type I Arch

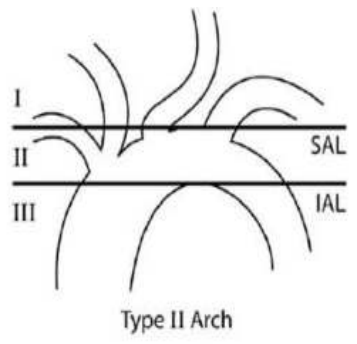

Type II Arch

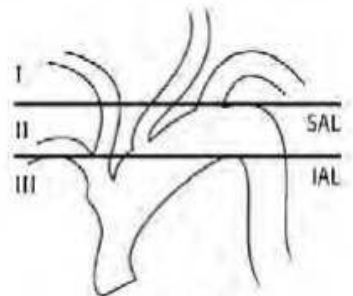

Type III Arch

Fig. Carotid 2: Schematic classification of aortic arch (3).

Above figures are quoted from reference 3

A: Type 1 arch: The origins of the great vessels to be catheterized are at the level of the superior arch line

B: Type 2 arch: The origins of the great vessels to be catheterized are between the superior and the inferior arch line

C: Type 3 arch: The origins of the great vessels to be catheterized below the level of the inferior arch line.

Type 1

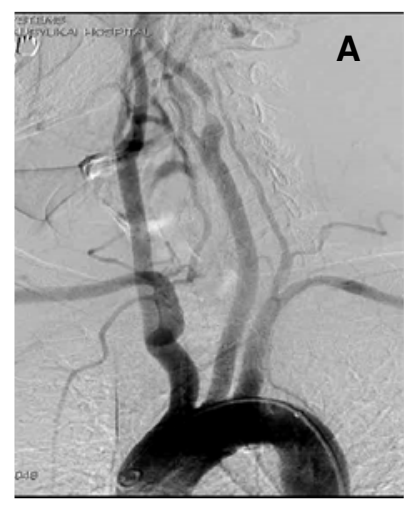

Type 2

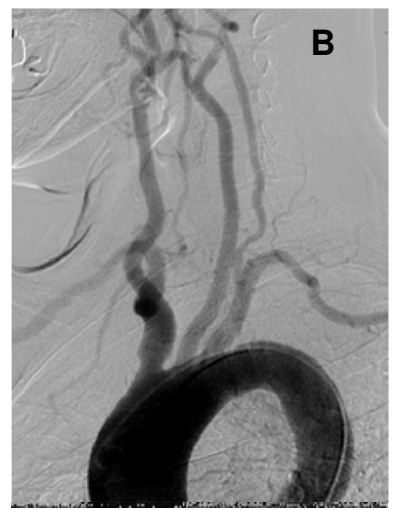

Type 3

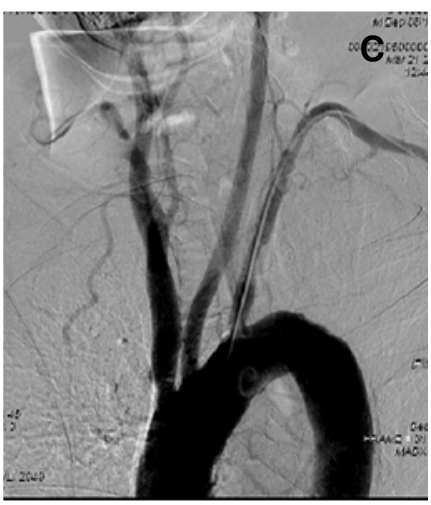

Fig. Carotid 3: Angiographic classification of aortic arch In Type 1(A) and Type 2(B), catheter can be easily selected. In Type 3(C), steep curve between the top of aortic arch and the origin of arch vessel is shown. This type 3 arch is difficult to negotiate to access to common carotid artery

The anomalies of bovine arch can frequently occur and may lead to prolonged fluoroscopy time to select left common carotid artery (Fig. Carotid 4). Fig. Carotid 4 A and B are the typical bovine arch. Access to left common carotid artery is easy in Fig. Carotid 4A, but In Fig. Carotid $4 \mathrm{~B}$, selection to left common may be difficult due to the sharp bend from top of aortic arch Most of the type 3 aortic arch is shaggy and cannulation of selective catheter carries the risks of embolization (Fig. Carotid 5). Even in type 1 aortic arch, horizontally angulated left common carotid artery makes it difficult to engage the catheter from transfemoral approach (Fig. Carotid 6). In recent multi-detector CT angiography, the similar information of aortic arch can be obtained (Fig. Carotid 7). 
Since an aortic arch angiogram or CT angiography can accurately reveal aortic arch type, complex anatomic variations and angulated takeoff. The assessment of aortic arch type is important for the success of CAS.
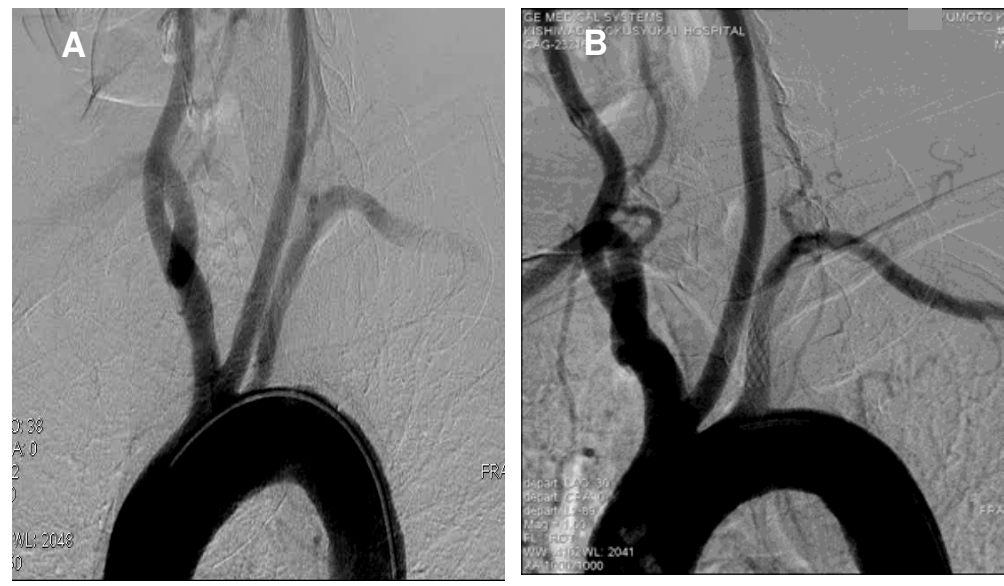

Fig. Carotid 4: Bovine arch

$A$ and B are the typical bovine arch. Access to left common carotid artery is easy in A, but In $B$, selection to left common may be a difficult due to the sharp bend from top of aortic arch
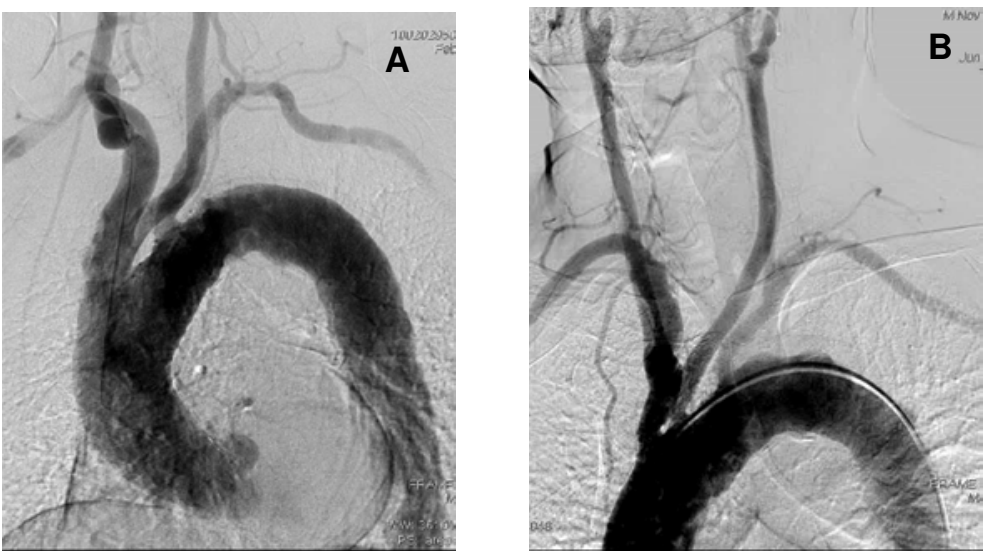

Fig. Carotid 5: Type 3 arch

A and B are typical type 3 arch. Cannulation of catheter to common carotid artery is difficult. Manipulation in these shaggy aortic arch carries the risks of embolization and a predictor of periprocedural complications. 


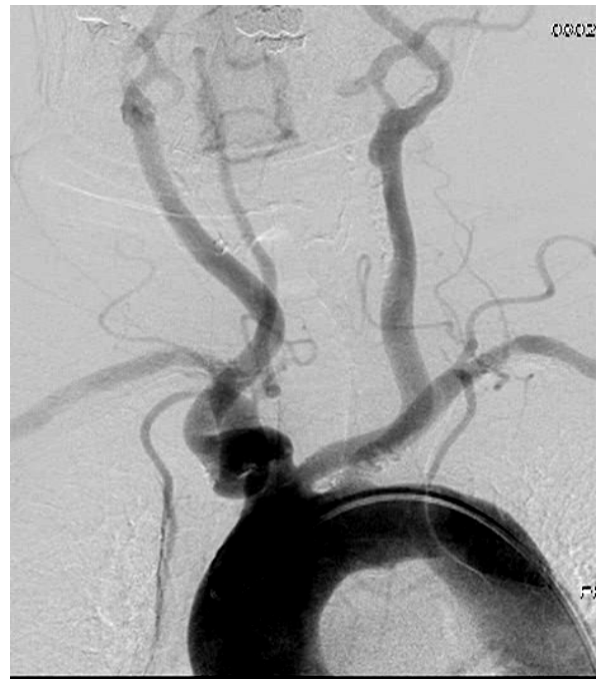

Fig. Carotid 6: Difficult aorta of type 1

Even in type 1 aortic arch, horizontaly angulated left common carotid artery makes it difficult to engage the catheter from transfemoral approach

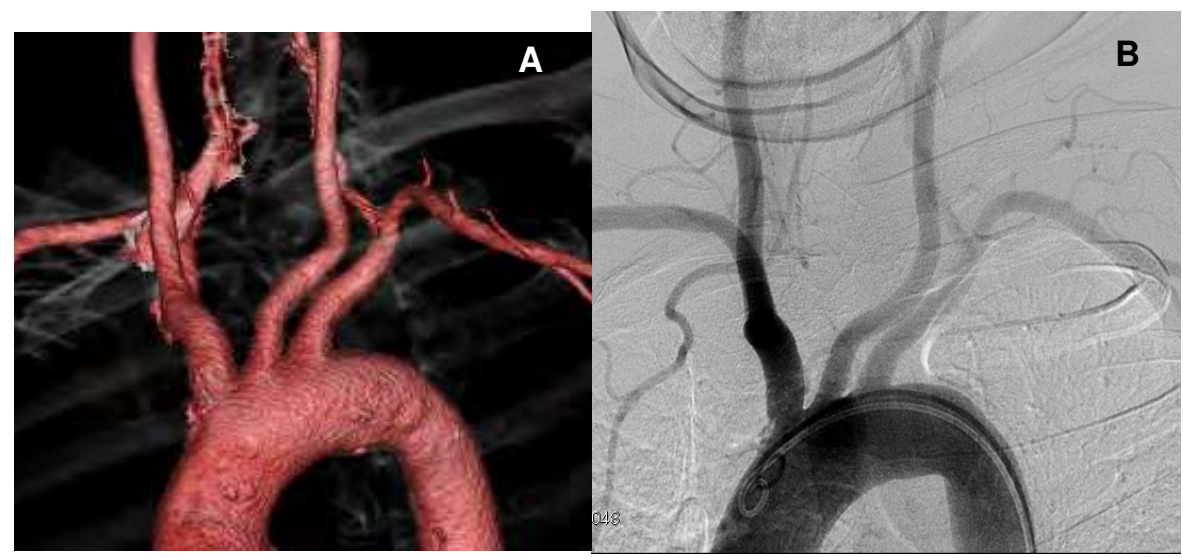

Fig. Carotid 7: Aortic arch assessment by 3D CT angiography and aortic arch angiography Aortic arch angiogram on the same patient. Similar aortic arch assessment can be obtained on either CT angiography (A) or angiogram(B)

\subsection{Selective carotid angiography}

\subsubsection{CTA vs. DSA}

To evaluate carotid stenosis, selective carotid angiography remains the golden standard. But multidetector CT angiography is rapidly becoming the preferred examination for the initial evaluation of carotid artery stenosis (4). CT angiography correlates to DSA. CTA (Fig. Carotid 8A) and DSA (Fig. Carotid 8B) images of left carotid stenosis were compared. 
In both images, tight stenosis were seen but CTA overestimates the stenosis. In calcified lesion, CTA does not reveal the real lumen. In Fig.8C,D, CTA image does not clarify the stenosis due to calcification.
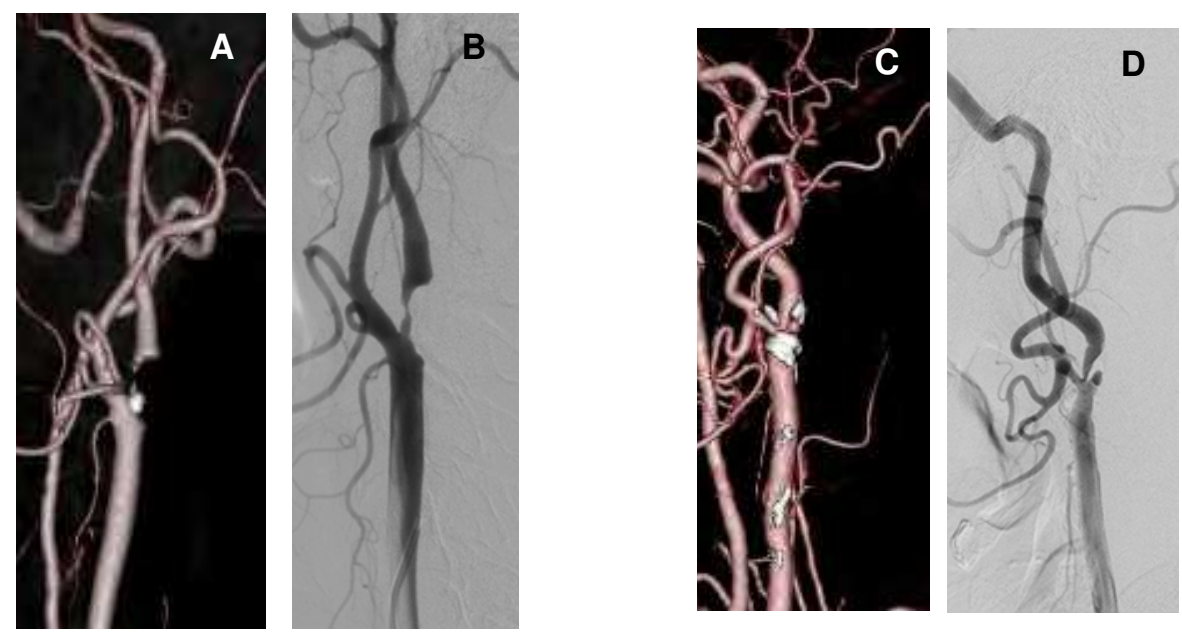

Fig. Carotid 8: Comparison of carotid artery stenosis between CT angiography and angiography

Left internal carotid artery (ICA) stenosis by CT angiography (A) was confirmed by digital subtraction angiography (B). CT angiography overestimates the stenosis.

In calcified lesion, CT angiography failed to show the ICA stenosis $(C)$ and DSA revealed the ulcerated ICA stenosis (D).

\subsubsection{Quantitative stenosis measurement}

The percentage of stenosis is expressed using the NASCET criteria (5). The NASCET criteria of $70 \%$ stenosis is the indication for CEA in symptomatic carotid stenosis. In NASCET, the stenosis calculated as the ratio of the diameter at the narrowest point to the diameter point at which beyond the area of post stentic dilatation. Before intervening carotid artery stenosis, quantitative angiographic assessment is required to measure the stenosis. So called NASCET measurement causes some confusion about the reference point of distal internal carotid artery. To avoid this confusion, minimal lumen Diameter (MLD) should be measured in mm (Carotid 9). In this case, \% stenosis based on NASCET was calculated at $87 \%$ with MLD $0.7 \mathrm{~mm}$.

\subsubsection{Angiographic view for carotid artery stenosis}

Basic angiographic view for carotid bifurcation can be obtained by anterior-posterior and lateral view. In most of the cases, lateral view shows the stenosis (Fig. Carotid 10). To indicate CAS or CEA, angiographic significant stenosis has to be found in multiple views. In these circumstances, 3D angiography is employed. In Fig Carotid 11, moderate stenosis was seen on either anterior or lateral view. In 3D angiography, right anterior oblique view at $60^{\circ}$ could reveal the tight stenosis. 


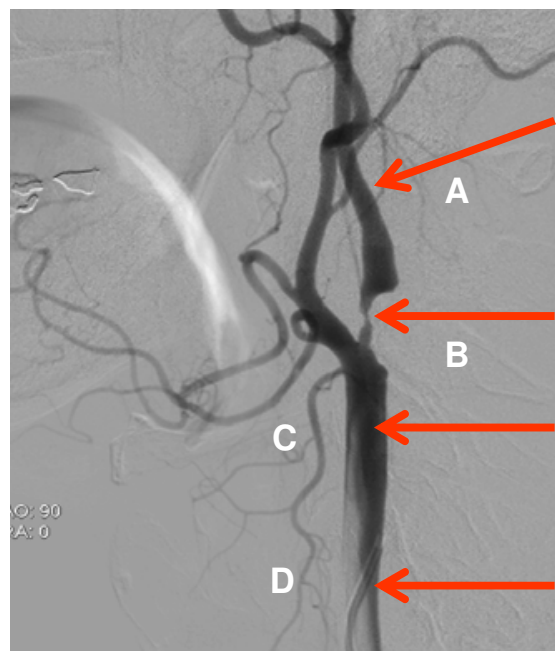

$5.2 \mathrm{~mm}$

$0.7 \mathrm{~mm}$

$8.3 \mathrm{~mm}$

$2.3 \mathrm{~mm}$

Fig. Carotid 9: Quantitative stenosis measurement

A: Distal internal carotid artery (ICA)

B: minimal lesion diameter (MLD)

C: Common carotid artery (CCA)

D: Reference catheter outer diameter

MLD should be express in mm. Distal ICA, MLD and CCA with reference catheter outerdiametr are needed to caluculate stenosis.
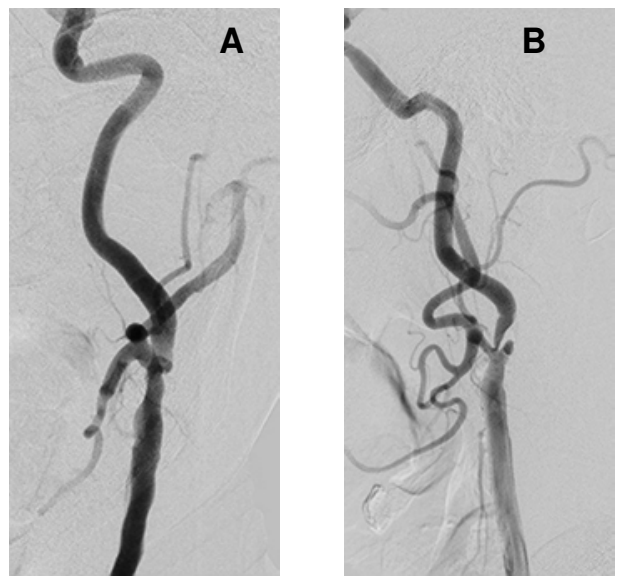

Fig. Carotid 10: Antero-posterior amd lateral view of left carotid artery bifurcation A: Antero-posterior view

B: Lateral view

Basic angiographic view for carotid bifurcation can be obtained by anterior-posterior (A) and lateral view(B). In most of the cases, lateral view shows the bifurcation stenosis 
A

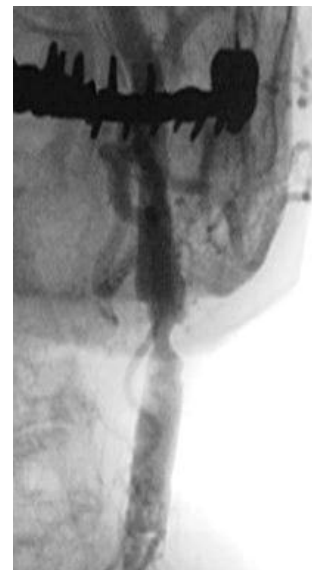

$\mathrm{B}$

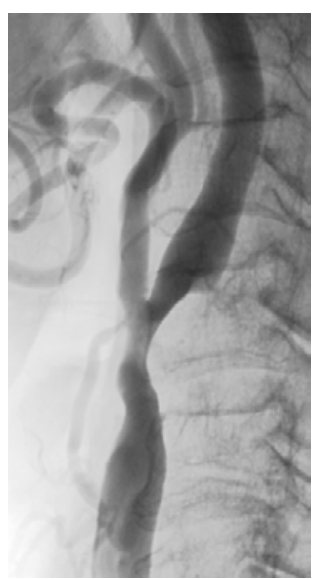

C

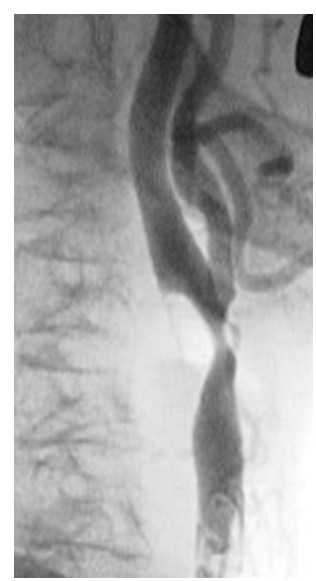

Fig. Carotid 11: 3D angiography for carotid artery stenosis assessment

A: Anterior view

B: Lateral view

C: Right anterior oblique $60^{\circ}$ view

Moderate stenosis were seen on either anterior or lateral view. In 3D angiography, right anterior oblique view at $60^{\circ}$ could see the tight stenosis.

\subsubsection{Level of carotid artery bifurcation and lesion}

Carotid artery bifurcates at the level of around C4. To indicate CAS, either anatomical or clinical high risks for CEA have to be clarified. One of the common indications is high position of bifurcation. The level of bifurcation or lesion level must be clearly demonstrated by lateral view. In Fig. Carotid 12A, bifurcation level is normal at the level of C4 In Fig. Carotid 12B, bifurcation is at the C3, but lesion extends to C2. In Fig. Carotid 12C shows the unusually low bifurcation.

\subsubsection{Plaque morphology}

Carotid artery plaque is evaluated by ultrasound or MRI, but angiogram also could show the large plaque burden (Fig. Carotid 13). Ulcer is commonly seen and the main source of cerebral emboli (Fig. Carotid 13A). Severe long stenosis is seen in Fig. Carotid 13B. This suggests the large plaque burden and CEA is recommended. Severe tight stenosis shows the string sings (Fig. Carotid 13C). This is the near occlusion and shows the sluggish antegrade flow. Real vessel size of distal ICA cannot be determined. In these cases, CEA is better indicated than CAS for thinking of complex plaque morphology.

\subsubsection{Tortuosity of ICA}

Protection device is placed at distal ICA or petrous portion. Atherosclerotic ICA sometimes shows the tortuosity. To place the protection device by the filter, the landing zone that was relative straight and $3 \sim 4 \mathrm{~cm}$ away from lesion site must be found. If there is no lamding 
A

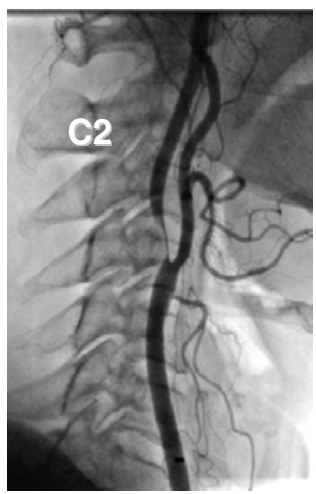

B

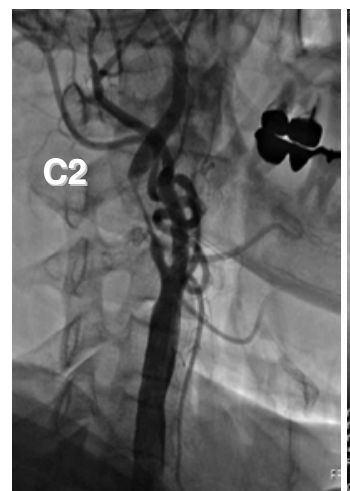

C

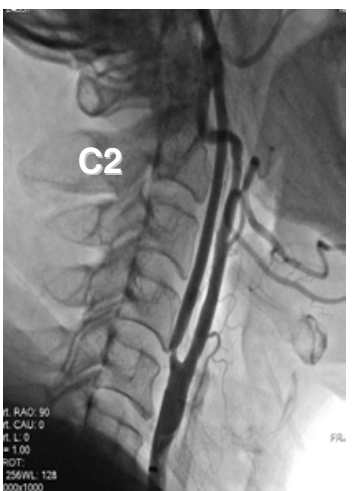

Fig. Carotid 12: Level of bifurcation and lesion A: Bifurcation level is normal at the level of $C 4$ $\mathrm{B}$ : Bifurcation is at the level of $\mathrm{C} 3$, but lesion extends to $\mathrm{C} 2$. C: Low bifurcation.
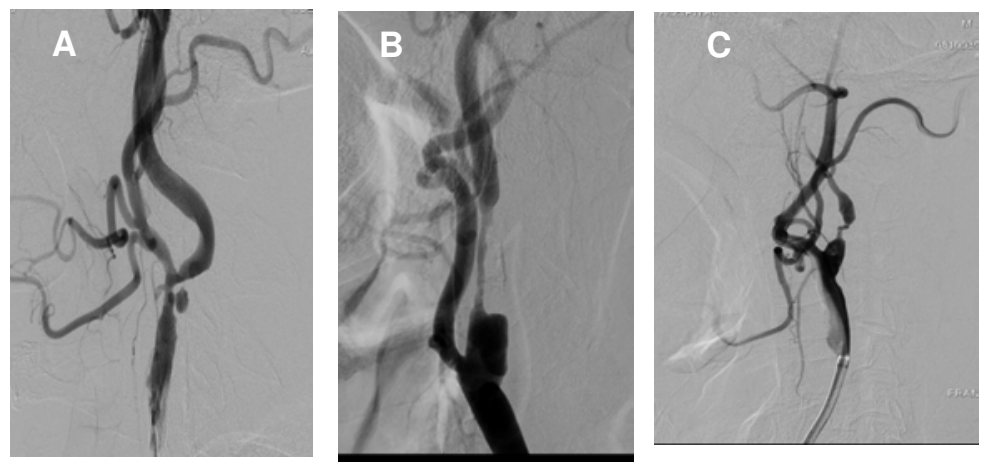

Fig. Carotid 13: Plaque morphology

A: Typical ulcer.

$\mathrm{B}$ : Severe long stenosis is seen and this suggests the large plaque burden

$\mathrm{C}$ : String sing is the preocclusion and shows the sluggish antegrade flow.

zone for filter, we have to consider proximal protection or balloon occlusion. In Fig. Carotid $14 \mathrm{~A}$, there is a landing zone but thinking of stent distal and filter position could be a very close and have the risks of filter trouble. In Fig. Carotid 14B, lesion show the directed to horizontally and upward bending of distal ICA. In this case, conformable stent is desirable and there is a risk of filter retrieval. In Fig. Carotid 14C, ICA shows the extreme tortuosity and CAS shoul be abandoned. 

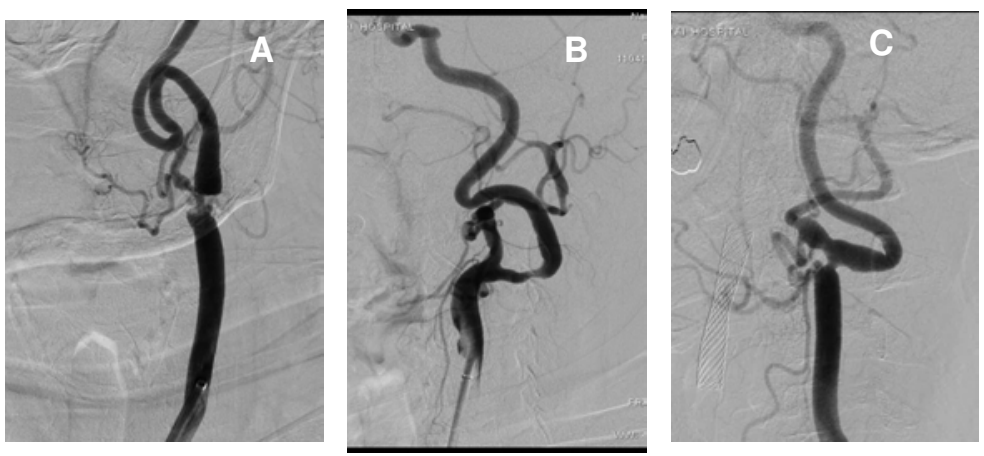

Fig. 14: Tortuosity of internal carotid artery (ICA)

A: $S$ curve of ICA.

B: 90 degree rightward shift of proximal ICA.

C: Marked tortuosity of ICA

These cases suggest carotid artery stenting are not a good indication for anatomical reasons.

\subsection{Summary}

Internal carotid artery stenosis is an important cause of ipsilateral stroke. CAS is becoming a valid alternative to CEA. Therefore angiography should be taken for thinking of CAS can be possible or not. Precise angiographic stenosis assessment, lesion location and lesion morphology are suitable for CAS. And also, place for filter landing zone have to be taken account.

\section{Subclavian artery angiography}

Occlusive disease of the supra-aortic trunks still remains an angiographic challenge. Among the proximal supra-aortic trunk disease, atherosclerosis is the most common cause of large artery occlusive disorder in the upper extremity.

In this chapter, angiographic approach for left subclavian artery disease will be discussed.

\subsection{Aortic arch angiography for branch disease}

Branch disease is defined of innominate, left common carotid, left subclavian artery (SCA) disease. In angiographic assessment of aortic branch disease, left anterior oblique (LAO) projection of aortography is the basic view. However, bony structure of thorathic cage and calcification of aorta made it difficult to obtain the clear image of arch. Severe proximal stenosis of three aortic arch vessels caused by atherosclerosis was shown in In Fig. SCA 1B, proximal left common carotid artery is stenosed. Stenosis or occlusion of innominate and ostium of left common carotid artery are not suited for revascularization by endovascular technique (6). In three aortic arch branch, left SCA stenosis most commonly seen and can be intervened by endovascular approach (Fig. SCA 1C). Atherosclerotic right SCA stenosis is mostly located at the ostium at the bifurcation of right common carotid artery. Aortography by LAO view showed right subclavian artery stenosis at the ostium (Fig. SCA 2A). Selective innominate artery angiography by right anterior oblique (RAO) view reveals that stenosis is located at the bifurcation of right common carotid artery (Fig. SCA 2B). Angioplasty to the right SCA ostium might affect right common carotid artery ostium. Extra caution is needed 
for right SCA ostium intervention, such as distal protection for right carotid artery territory. Among the three aortic arch branch vessels, atherosclerotic left SCA is commonly found in proximal to vertebral artery and left SCA is most favourable fort endovascular therapy. In this chapter, left SCA angiogram for interventional approach is discribed.
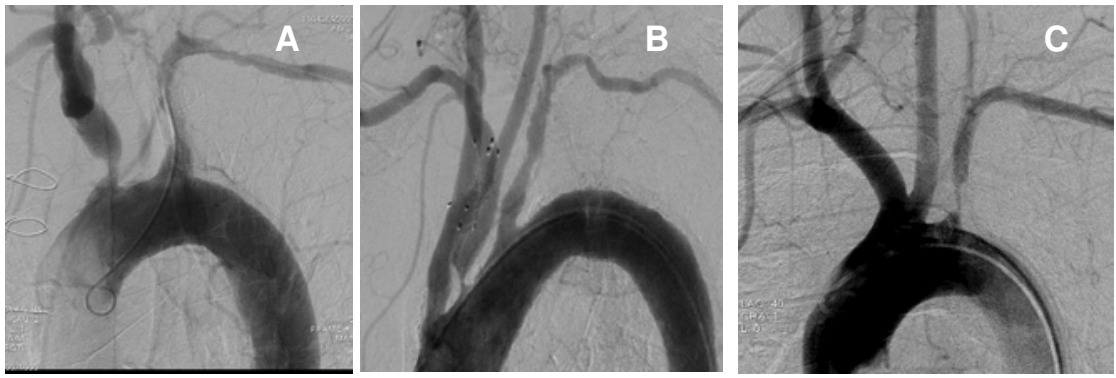

Fig. SCA 1: Aortic arch angiogram of aortic branch disease

A: Innominate, left common, left subclavian artery are diseased.

B: A tight ostial stenosis of left common carotid artery in type 3 arch

C: Typical left subclavian artery stenosis. Bovine arch is noted.
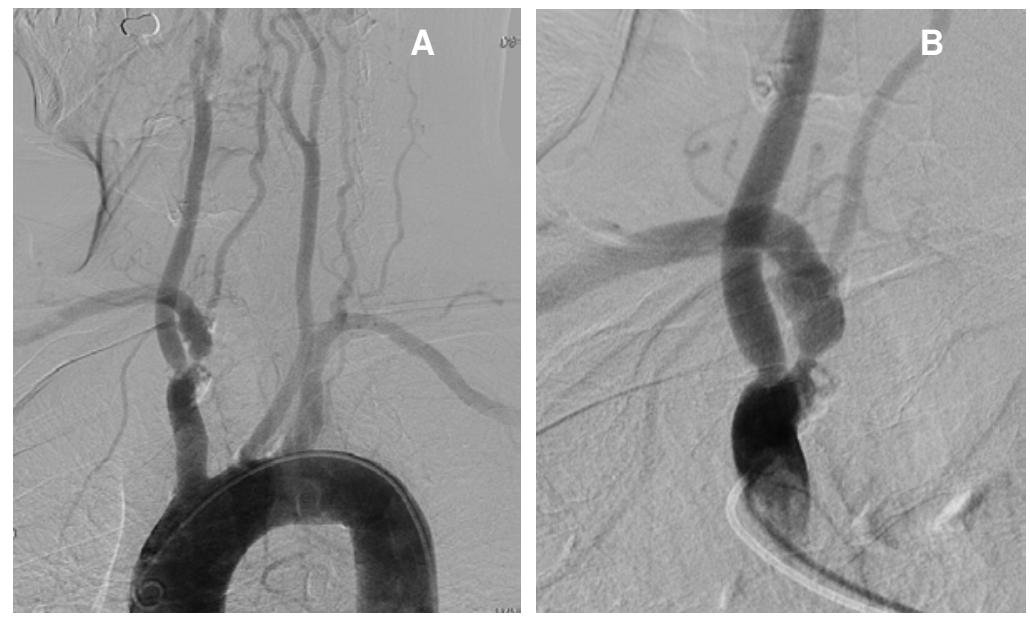

Fig. SCA 2: Ostial location of right subclavian artery stenosis

A: Aortography by left anterior oblique view showed right subclavian artery stenosis at the ostium

B: Selective innominate artery angiography by right anterior oblique view reveals that stenosis is located at the bifurcation of right common carotid artery.

\subsection{Aortic arch angiogram for left subclavian artery stenosis}

In subclavian artery angiography, nonsubtracted image gives the anatomical information but does not show the detail of subclavian artery stenosis (Fig. SCA 3A). Digital subtraction angiography (DSA) clearly delineates subclavian artery from the background (Fig.SCA3B). 


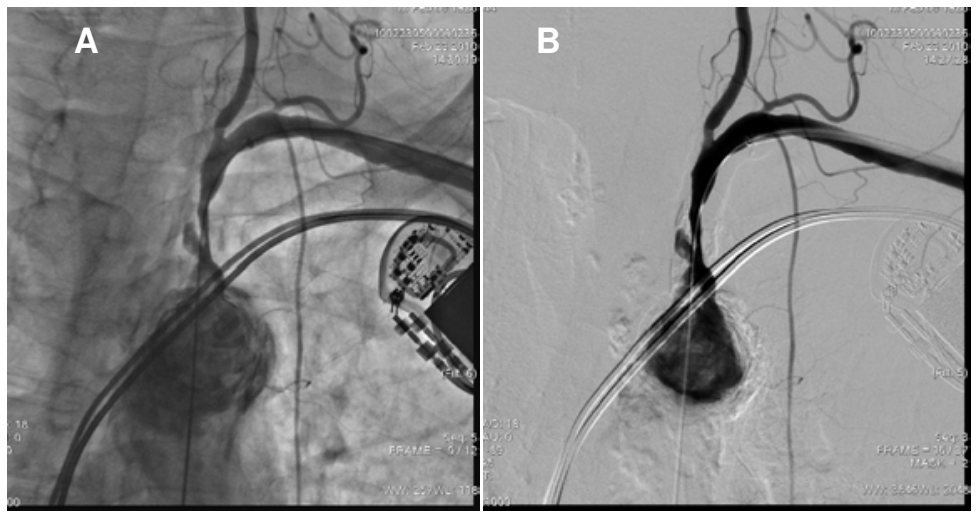

Fig. SCA 3: Non-substracted and subtracted angiogram of left subclavian stenosis A: Non subtracted angiography gives the anatomical information B: Digital subtraction angiography (DSA) clearly delineates subclaviar artery stenosis

As the initial angiographic approach for left SCA stenosis or occlusion, aortic arch angiography should be taken in left anterior oblique (LAO) $30 \sim 45^{\circ}$ view by DSA (Fig. SCA 4A). Contrast volume and speed are at least $18 \mathrm{cc} / \mathrm{s}$, total $20 \sim 30 \mathrm{cc}$ by using 5Fr Pigtail catheter. In Fig. SCA 4A the image was taken by $30 \mathrm{~cm}$ in size and delayed image reveals the distal SCA via collateral from right vertebral artery (Fig. SCA $4 \mathrm{~B})$. Selective right vertebral angiogram proves the reversed flow of left vertebral artery through basal artery (Fig. SCA4C). Image size of aortic arch angiography is usually taken by $30-40 \mathrm{~cm}$ image (Fig. SCA $5 \mathrm{~A}$ ). Thinking of interventional approach, $20 \mathrm{~cm}$ image is more practical to cannulate catheter to left SCA (Fig. SCA 5B). But to confirm the exact location of stenosis, selective left subclavian artery angiogram is required (Fig. SCA 5C). In Fig. SCA 6A, aortogram showed the total occlusion of proximal left SCA, but
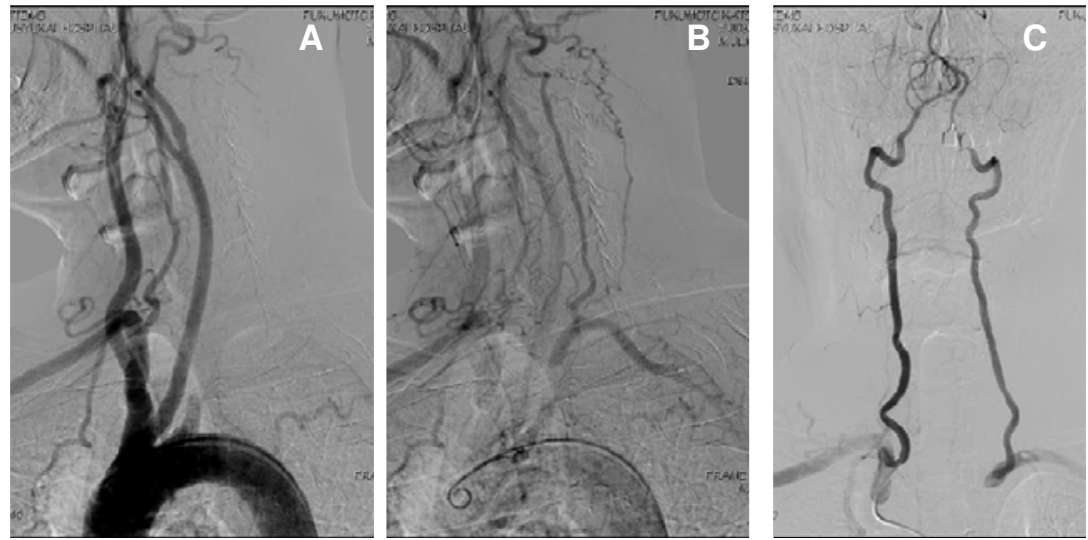

Fig. SCA 4: Angiogram of left subclavian artery occlusion

A: Aortography by LAO $45^{\circ}$ by $30 \mathrm{~cm}$ image size

B: Delayed image reveals the distal SCA via a collateral from right vertebral artery

C: Selective right vertebral angiogram proves the reversed flow of left vertebral artery through basal artery 
selective injection revealed the $95 \%$ stenosis (Fig. SCA 6). To determine subtotal or occlusion, selective angiography is needed.

$30 \mathrm{~cm}$

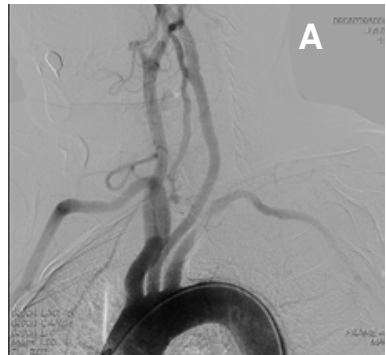

$20 \mathrm{~cm}$

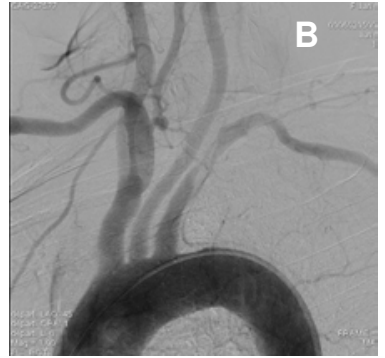

selective

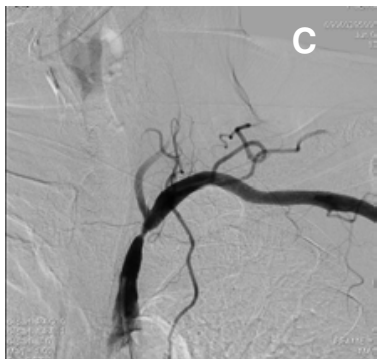

Fig. SCA 5: Angiographic image size of for left subclavian artery disease A: Aortic arch angiography is usually taken by $30 \mathrm{~cm}$ image

B: To cannulate catheter selectively to left subclavian artery, $20 \mathrm{~cm}$ image of angiogram is appropriate

C: Selective left subclavian artery angiogram is needed to confirm the stenosis.
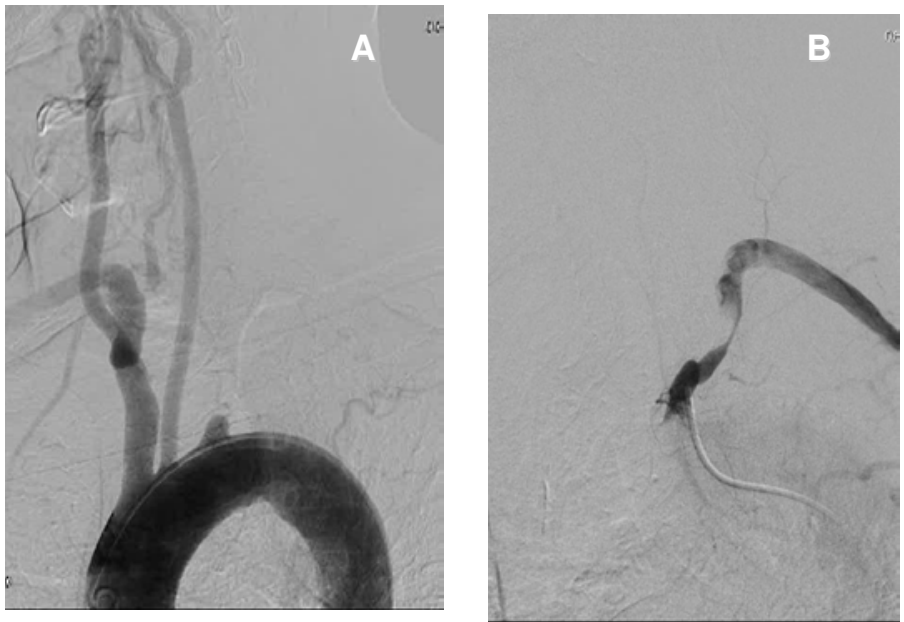

Fig. SCA 6: Aortography and selective angiography

A: Aortagram showed the total occlusion of proximal left subclavian artery B: Selective left subclabian artery angiogram showed subtotal stenosis.

Selective angiography is necessary to intervene the lesion. 


\subsection{Angiography for subclavian artery intervention.}

Although there is a paucity of long-term data of endovascular therapy, subclavian artery stenting is now the standard approach and offer many advantage over surgery in terms of morbidity and mortality. To succeed in left SCA stenting, a precise location of stenosis or occlusion must be visualized.

Fig. SCA 7 shows the typical left SCA stenosis. Initial aortogam by LAO view reveals left SCA stenosis (Fig. SCA 7A). To intervene left SCA stenosis, stenosis should be exactly located. Selective angiography combined with brachial artery catheter injection of dye clearly demonstrates the tight stenosis of left SCA (Fig. SCA 7B). Based on this angiogram, further stenting became the straightforward procedure (Fig. SCA 7C). The simultaneous injection of dye from distal and proximal SCA is very useful technique for subclavian artery intervention. To confirm SCA occlusion, the similar technique is applied during aortography. Selective SCA angiogram by injecting dye through catheter from brachial artery at the time of aortography showed the exact occlusion site (Fig. SCA 8A). This kind of angiogram leads to the successful intervention. Same angiographic technique was taken after stenting (Fig. SCA 8B).
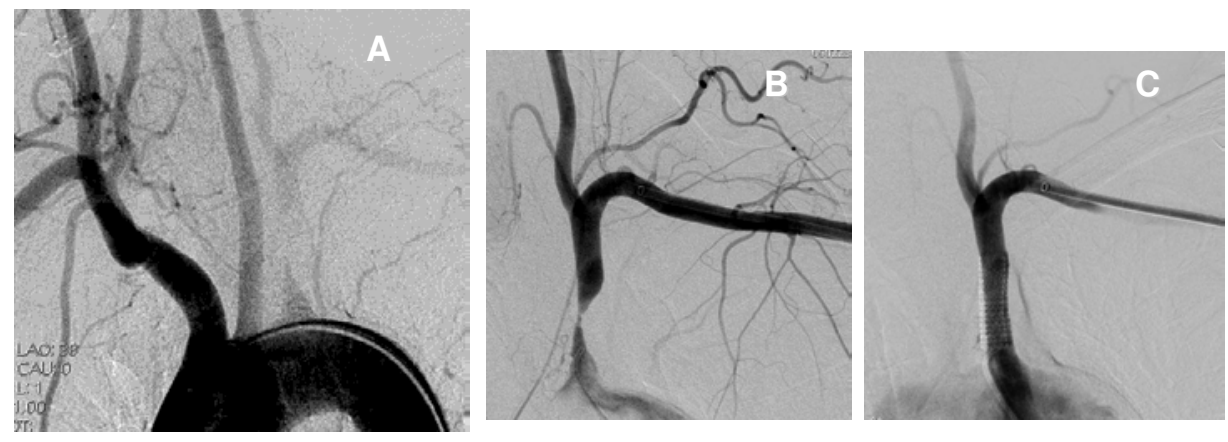

Figure. SCA7: Angiographic technique to assess left subclavian stenosis

A: Initial aortagram by LAO view reveals left SCA stenosis

B: Selective angiography combined with brachial artery catheter injection of dye clearly demonstrates the tight stenosis

C: Selective angiogram of post stenting. Distal and proximal injection of dye clearly demonstrate successful stent implantation

Not all angiogram could show the clear image of subclavian stenosis. In these cases, hemodynamic assessment is the useful to confirm a significant stenosis. In Fig. SCA 9A, simultaneous pressure recording was performed and showed $40 \mathrm{mmHg}$ peak systolic gradient and left SCA stenosis was located at the ostium. After stenting, no gradient was detected and successful stent placement was confirmed (Fig. SCA 9B).

\subsection{Angiographic assessment for coronary- internal mammary steal}

The increased employment of internal mammary artery (IMA) grafts for coronary revascularization, proximal SCA stenosis is becoming well known cause of coronary- 

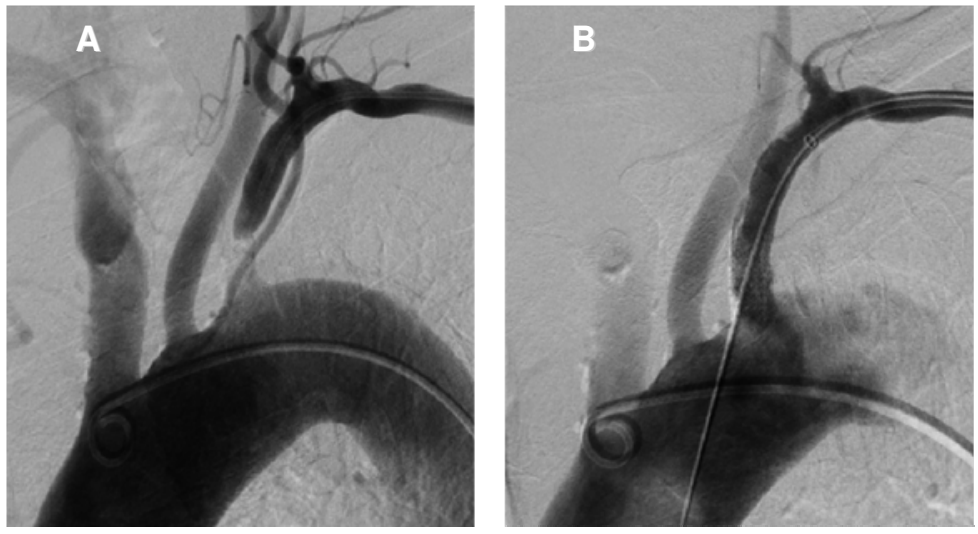

Fig. SCA 8: Angiographic technique for left subclavian occlusion

A: Aortography with simultaneous injection of dye through the catheter from brachial artery. This angiogram gives the precise morphologic information of occlusion.

B: Aortography of post stenting.

Same angiographic technique was taken and shows the successful stent placement.
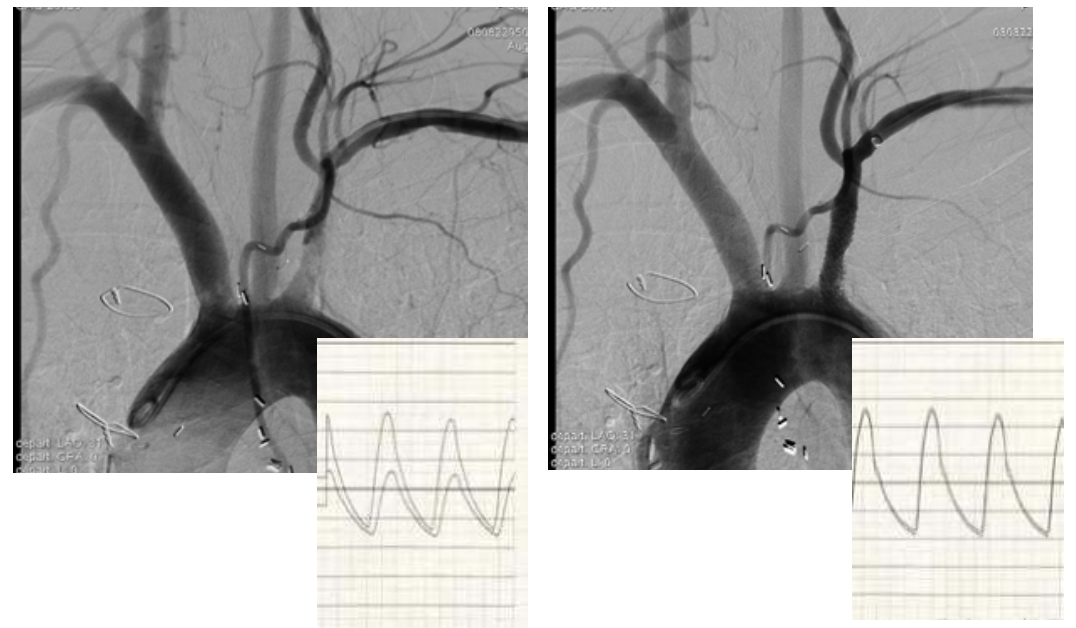

Fig. SCA 9: Simultaneous pressure tracing for subclavian artery stenosis

A: Aortography with selective distal SCA angiogram. Angiogram does not clearly reveal SCA stenosis. Simultaneous pressure tracing confirmed the siginificant stenosis.

B: Aortography of post stenting. Distal and proximal pressure were equalized and successful stenting was proven by hemodynamic study.

subclavial steal. Left SCA stenting is the good indication for coronary to left IMA steal (7). In coronary steal to left IMA, selective left coronary angiogram is needed to prove reversed flow of IMA. The 70-year-old patient with suspected coronary steal was shown in Fig. SCA 10A. The left coronary angiogram showed the typical coronary steal which left IMA shows 
reversed flow and draining into left SCA is seen. This image was taken by coronary mode, but point is to prove coronary to left SCA steal phenomenon and DSA image can be used with $30 \mathrm{~cm}$ image (Fig. SCA10B).
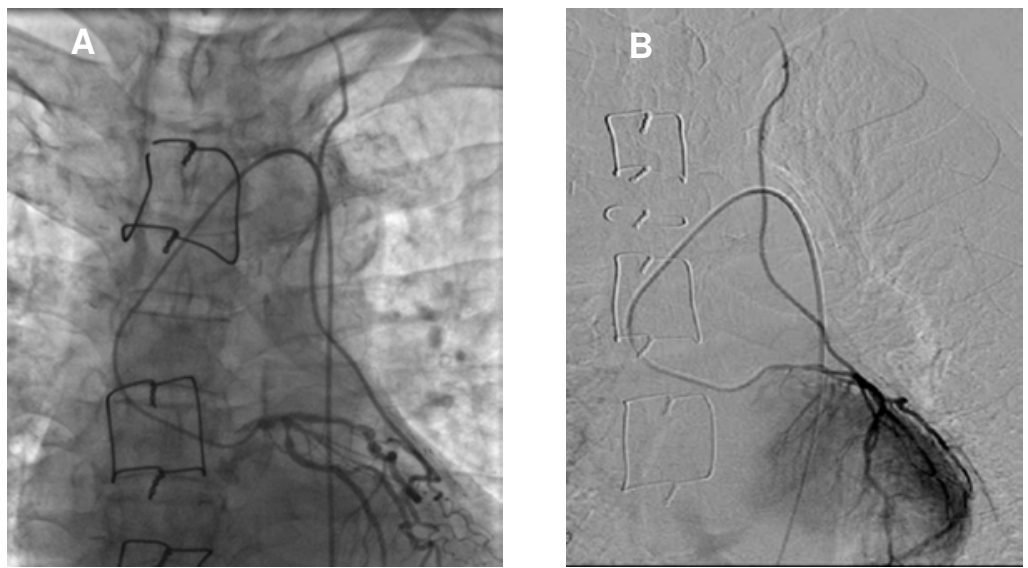

Fig. SCA10: Left coronary angiography for coronary-subclavian steal

A: Suspected coronary steal patient. Left coronary angiogram showed revealed reversed flow of left IMA. This angiogram was taken by coronary mode.

B: Similar left coronary angiogram was taken by digital subtraction angiography. IMA is more well visulalized. Either method can be used to prove IMA to left SCA. $30 \mathrm{~cm}$ of image is preferable.
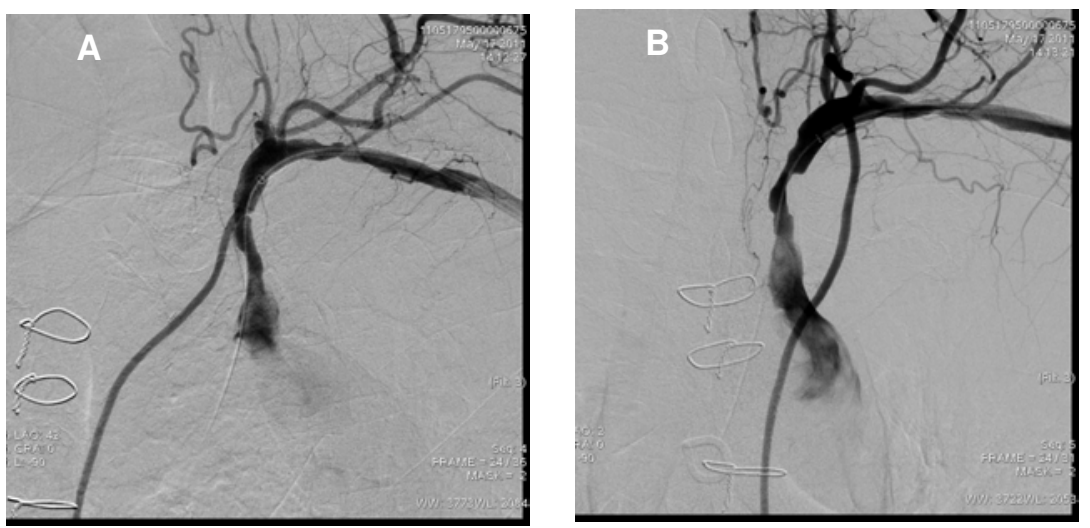

Fig. SCA 11: Relation between proximal left subclavian artery stenosis and origin of left internal mammary artery (IMA)

A: Left subclavian artery angiogram by left anterior oblique (LAO) view. Origin of left IMA is not identified by LAO view.

B. Left subclavian artery angiogram by antero-posterior view.

Relation between left IMA and proximal SCA stenosis is well seen. 
Most of the initial left SCA angiogram is taken by LAO view. However, in this view, in some cases, left IMA origin is not well seen (Fig. SCA 11A). Relation between left SCA stenosis and IMA is very important for stent placement. The left SCA selective angiogram was taken by anterior-posterior (AP) view (Fig. SCA 11B). In AP view, origin of left IMA is visualized and relation between left SCA stenosis and IMA is well understood. This could lead to successful stent placement.
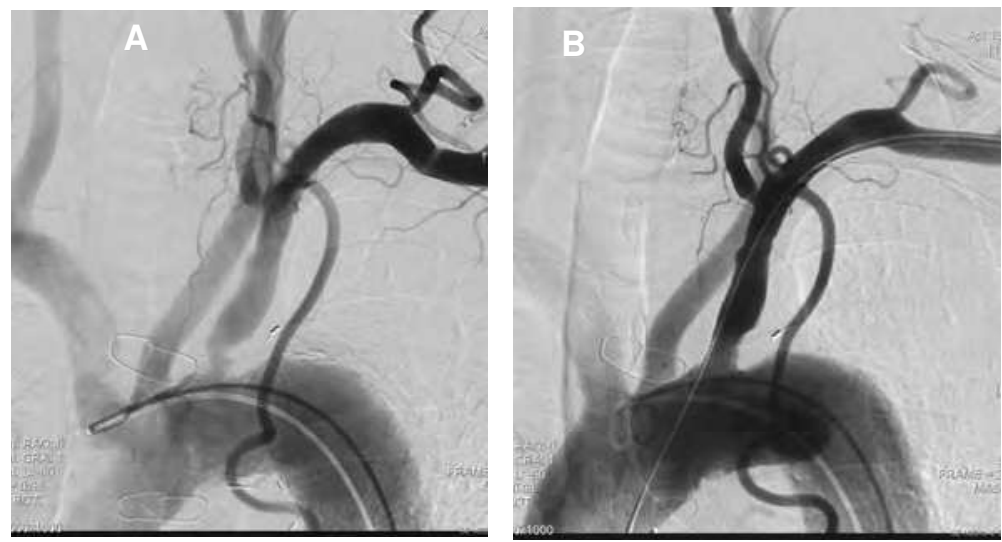

Fig. SCA12: Pre and post left subclavian artery stenting in coronary-subclavian steal A: Aortic arch angiogram.

A 65 year-old female post coronary bypass patient, lesion was located at left SCA ostium. B: Aortic arch angiogram post stenting. This angiogram showed the successful stenting.

In left SCA ostium disease, precise lesion location is mandatory. In Fig. SCA 12, a 65 yearold female post coronary bypass patient, lesion was located at left SCA ostium. This was confirmed aortography combined with brachial side simultaneous injection of dye (Fig. SCA 12A). Based on the angiogram, stent was precisely implanted and same angiographic technique was repeated to confirm successful procedure.

\subsection{Summary}

Primary stenting for a symptomatic SCA stenosis can be performed with relatively safe procedure risks. However, to succeed SCA stenting, the stenosis or occlusion must be clearly visualized by angiography. Aortic arch angiography with selective angiography by using distal injection of dye gives the precise lesion location and could lead to successful intervention. When patient presents after coronary bypass with coronary-subclavian steal, SCA stenting is the good option. But, origination of IMA must be precisely identified. Thereis no clinical randomized study about SCA stenting. To intervene to subclvian artery, a meticulous angiographic assessment is required.

\section{Renal artery angiography}

Atherosclerotic renal artery stenosis (ARAS) is an increasingly recognized cause of severe hypertension and declining kidney function (8). Typically involving the renal artery ostium or proximal segment of the renal artery. Patients with ARAS have been demonstrated to 
have an increased risk of adverse cardiovascular events (9). However, the efficacy of renal artery stenting for ARAS is a bit of controversial since ASTRAL trial was published (10). Discordance exists between the procedural success rate and the equivocal clinical response rate after renal stent placement, which is likely to be a result of poor patients selection and inadequate angiographic assessment of lesion severity. Angiographic technique for ARAS will be discussed in this chapter.

\subsection{Aortography for renal artery stenosis}

Aortography and selective renal artery angiography considered to be the gold standard for assessing renal artery anatomy and renal artery stenosis. 3D CT angiography consists of a continuously overlapping transaxial images and is now replacing aortography for the diagnostic purpose. The drawback of aortography, only one shot image can be obtained and the image may not be in a single plane to see the both renal artery ostium. We usually take left anterior oblique view $15 \sim 30^{\circ}$ for initial aortography (Fig. Renal 1). In Fig. Renal 1, bilateral renal artery stenosis is well visualized in one flame.

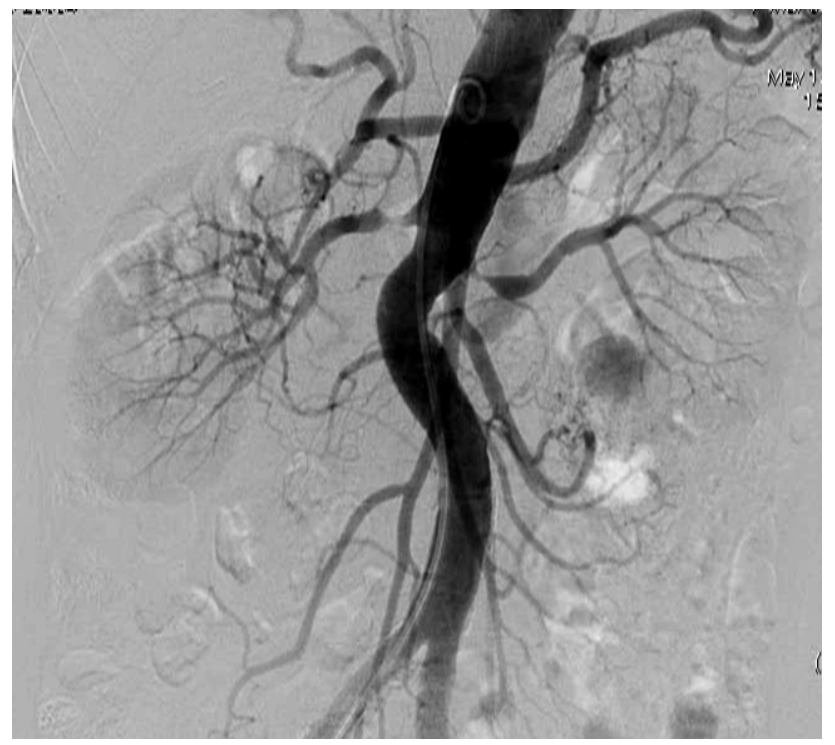

Fig. Renal 1: Aortography for the assessment of renal artery stenosis

Aortography was taken by left anterior oblique view $30^{\circ}$. Typical bilateral atherosclerotic renal artery stenosis is shown in one flame.

- Multiple renal arteries: Computed tomography angiography (CTA) with multiple detector-row CT (MDCT) has evolved into an established technique for imaging of renal and mesenteric vessels. Particularly, in multiple renal arteries, MDCT is superior to DSA to detect all renal arteries. To make a correct diagnosis of multiple renal arteries, MDCT and DSA could be used complimentary. In Fig. Renal 2, a case of multiple renal arteries are shown in both MDCT and DSA. In DSA, many other arteries were shown and we might miss to identify all 4 renal arteries. With the information of MDCT image, all renal arteries were confirmed. 

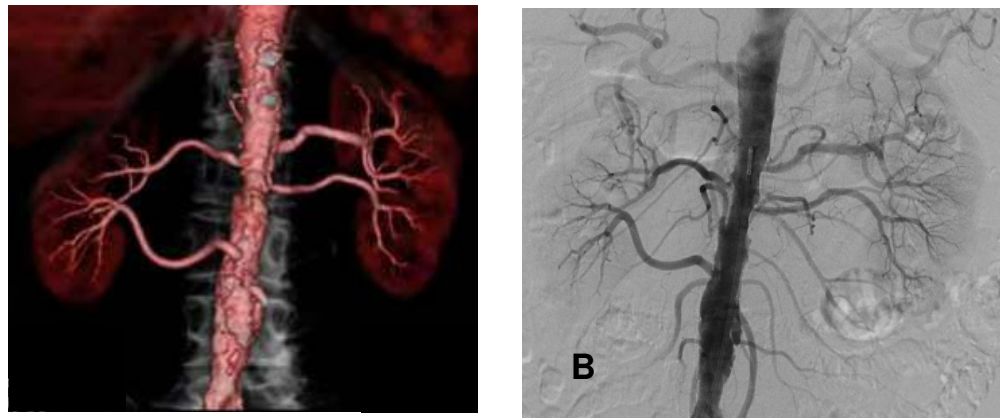

Fig. Renal 2: CT angiography and angiography for multiple renal arteries A: CT angiography showed two renal arteries on both kidney.

B: The same view was taken by angiography. 4 renal arteries were seen but many other arteries are included and might miss multiple renal arteries.

- Right renal artery ostium and super mesenteric artery: Often times, right renal artery overraps to superior mesenteric artery (SMA) by antero-posterior view and proximal right renal is not visualized (Fig. Renal 3A). Selective right renal artery angiography confirmed the tight stenosis in right renal artery ostium (Fig. Renal 3B). There are reports about the detection of renal artery stenosis by aortography at the time of coronary angiography. But in reality, the simple aortogram might not identify the stenosis of right renal artery proximal stenosis.
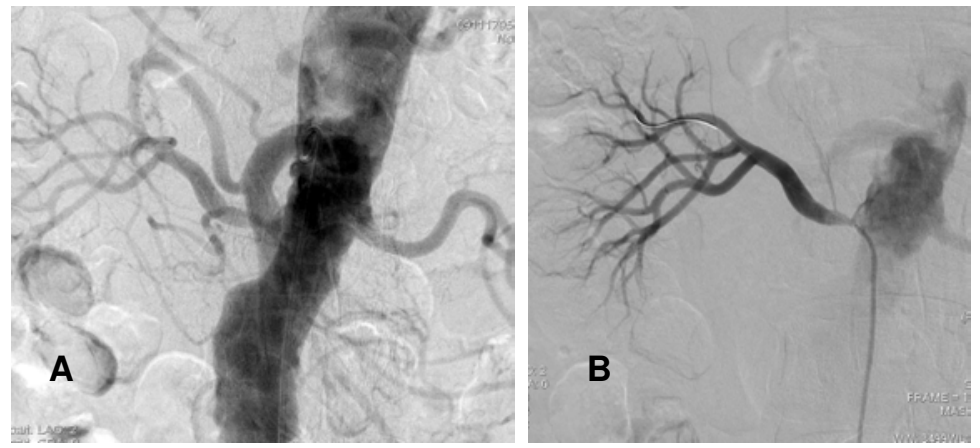

Fig. Renal 3: Relation between right renal artery ostium and super mesenteric artery A: In aortography of left anterior oblique view, right renal artery overraps to superior mesenteric artery and proximal right renal artery is not shown B: Selective right renal artery angiography confirmed tight stenosis in proximal right renal artery ostium

- Access for selective renal artery catheter placement: Aortography gives the important information about the access to selective renal artery angiography. The straight aorta with minimal atherosclerotic change is seen in Fig. Renal 4A. This suggests easy access for left renal catheter engagement. In Fig. Renal 4B, there is a marked tortuosity of aorta and tranfemoral approach may face difficulty to reach both renal artery ostium. In this case, transbrachial or transradial approach should be considered. 

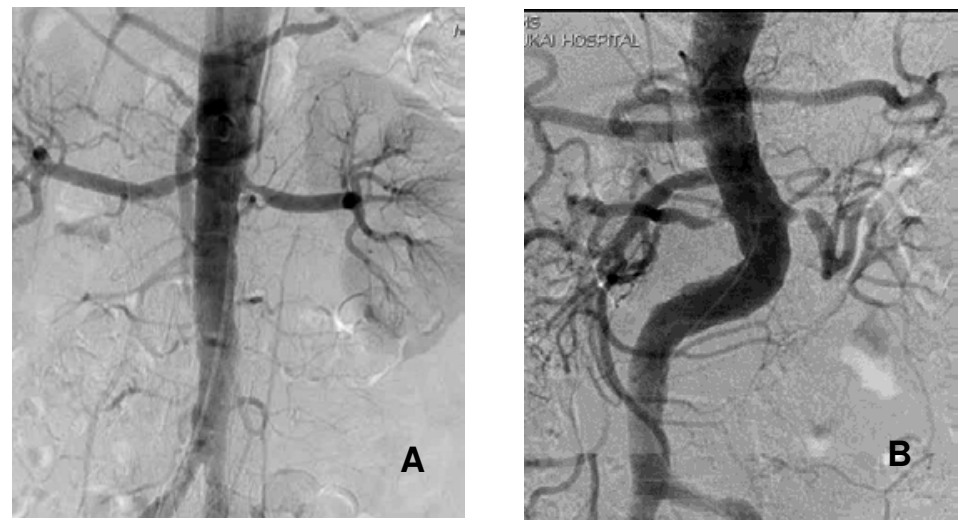

Fig. Renal 4: Access for selective renal artery catheter placement

A: The straight aorta with minimal atherosclerotic change is seen. This suggests easy access for left renal catheter engagement.

$\mathrm{B}$ : There is a marked tortuosity of aorta. Selective angiography by tranfemoral approach might be difficult procedure to reach both renal artery. In this case, transbrachial or transradial approach is recommended.

\subsection{Selective renal artery angiography}

Selective renal artery angiography is the definitive gold standard for the diagnosis of the significant renal artery stenosis. In aortography, clear relation between aorta and renal artery ostium is shown (Fig. Renal 5A). However, in aortography, information of intrarenal arteries is not obtained. Only selective renal artery angiography could show the picture of intrarenal artery as well as proximal stenosis (Fig. Renal 5B).
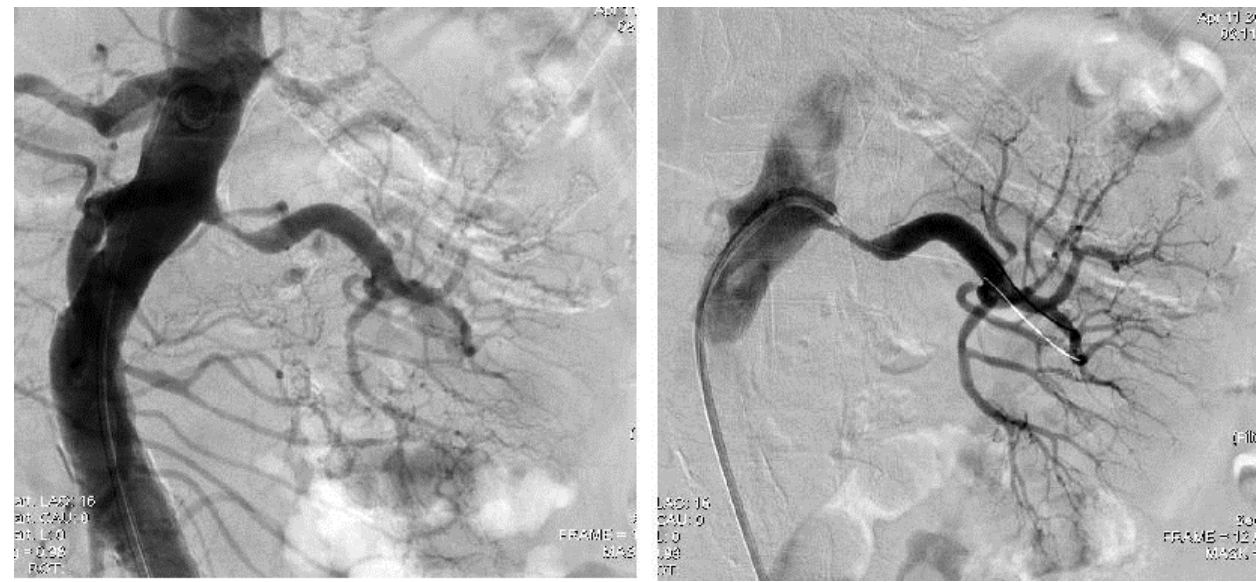

Fig. Renal 5: Aortography and selective renal artery angiography

A: Aortography shows the clear relation between aorta and renal artery ostium stenosis. B: Selective renal artery angiography could show precise renal artery stenosis and anatomy of intrarenal arteries. 


\subsubsection{Digital subtracted image}

By using digital subtraction angiography (DSA), the excellent renal artery angiography is Taken (Fig. Renal 6A). But in reality, it is very difficult to take excellent DSA image. Recent advances of digital image, distinction between subtracted and non subtracted image are relatively small (Fig. Renal 6B). Non DSA image is more important for renal artery intervention. In the near future, good quality digital image might replace DSA at least in renal artery.
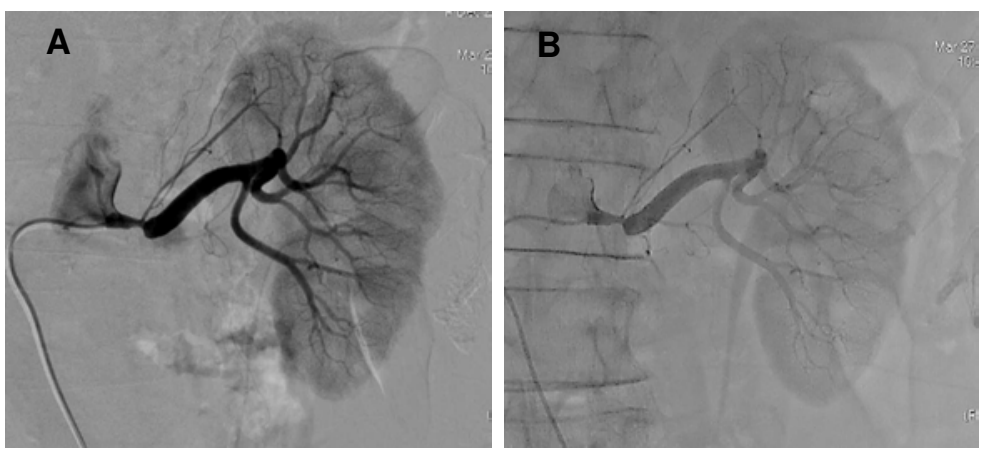

Fig. Renal 6: Digital subtracted and non subtracted selective renal artery angiography

A: Digital subtracted renal artery angiography.

B: Non subtracted image

Digital subtraction angiography (DSA) gives excellent image of renal artery vasculature(A). Recent advances of digital angiography shows that image quality between subtracted and non subtracted image are relatively small(B).

\subsubsection{Ideal renal artery angiography}

In selective renal artery angiography, catheter tip is in the renal artery ostium, but most of catheter goes into distal to stenosis and relationship between ostium and stenosis is not clarified. Aortagraphy of typical bilateral ARAS is shown in Fig. Renal 7A). In selective right renal artery angiography, catheter tip goes further to stenosis (Fig. Renal 7B) and relationship between ostium and stenosis is not clear. The ideal selective renal artery angiography is shown in Fig. Renal 8. In Fig. 8A, catheter tip attached to stenosis and is not recommended to inject dye. The ideal renal artery angiography is catheter tip located in aorta and could see the proximal stenosis (Fig. Renal 8B). To take the good quality renal artery angiogram, lesion is crossed by soft coil 0.014inc. wire. After confirming normal aortic pressure pattern, dye should be injected. If the catheter is too close to stenosis, slight drawback of catheter is needed to take the good quality angiogram. By doing this procedure, correct stenosis assessment can be made.

\subsubsection{Lesion location}

In ARAS, stenosis is basically localized in proximal renal artery. There are 3 types of lesion locations (11). One is typical ostial stenosis (Fig. Renal 9A). Lesion located in middle of the proximal renal artery is called renal type (Fig. Renal 9B). In most of the cases, mixed type of stenosis is seen (Fig. Renal 9C). 

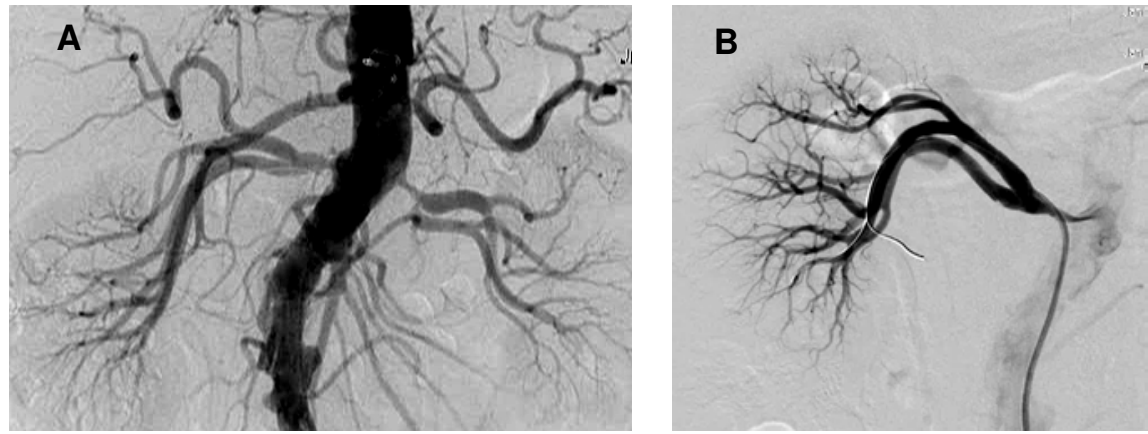

Fig. Renal 7: Catheter tip position of selective renal artery angiography A: Aortography of typical bilateral ARAS B: Selective right renal artery angiography. In selective angiography, catheter tip goes into further stenosis. Relation between ostium stenosis and aortic wall is not elucidated.
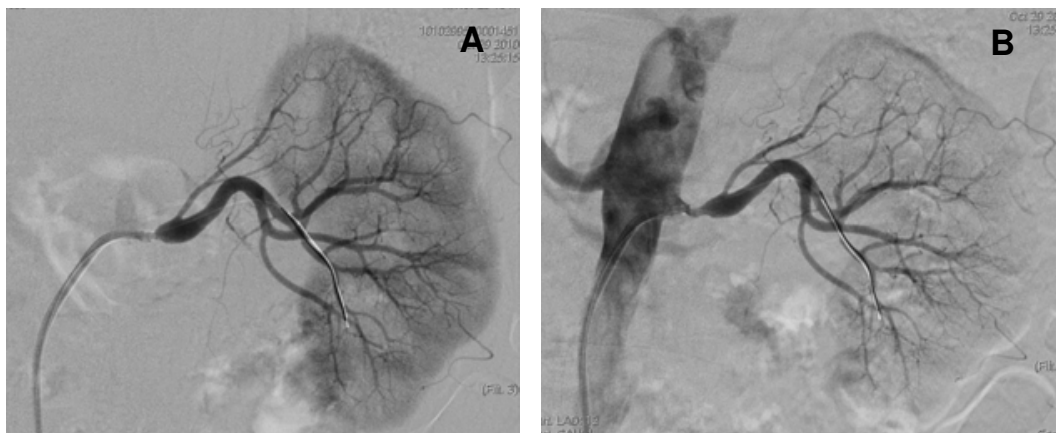

Fig. Renal 8: Ideal selective renal artery angiography

A: Catheter tip attached to stenosis and injection of dye at this place might damage the vessel.

B: The ideal renal artery angiography. Catheter tip is located in aorta and could see the proximal stenosis with distal renal vasculature.
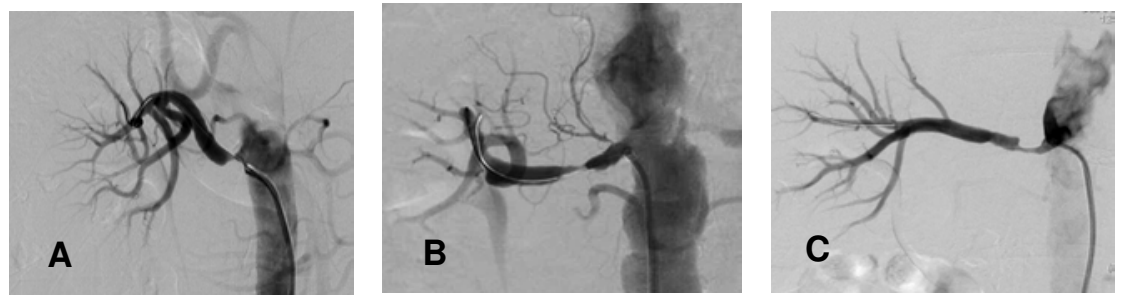

Fig. Renal 9: Lesion location of atherosclerotic renal artery stenosis. Atherosclerotic renal artery stenosis is basically localized in proximal renal artery. There are 3 types of lesion locations. One is typical ostial stenosis (A). Lesion located in middle of the proximal renal artery is called renal type(B). In most of the cases, mixed type of stenosis is seen (C). 


\subsubsection{Reference vessel}

To select the correct stent size, accurate vessel diameter must be measured (12). To make a correct assessment of stenosis and vessel size, reference point is chosen. In Fig. Renal 10A, reference vessel is considered to be about $2 \mathrm{~cm}$ distal to stenosis. However, in Fig. Renal 10B, post-stenostic dilatation is seen and bifurcation follows (Fig. Renal 10B). In this case, reference vessels cannot be determined. The bifurcation located at the ostium, real vessel size is not known (Fig. Renal 10C). This fact is not well understood and this is the main cause of recent confusion of stenosis evaluation.
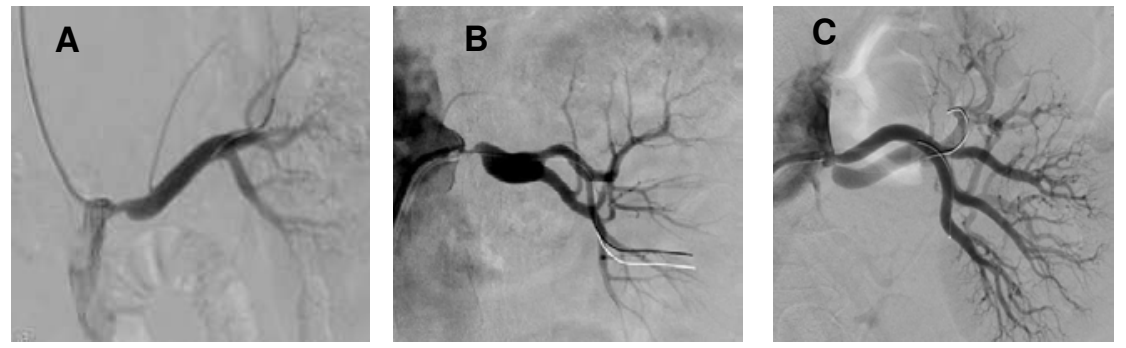

Fig. Renal 10: Reference vessel

To calculate \% diameter stenosis or minimal lesion diameter, reference vessel must be determined.

A: Reference vessel can be determined about $2 \mathrm{~cm}$ distal to stenosis.

B: Typical post-stenostic dilatation is seen and bifurcation follows. In this case, reference vessels can not be determined.

C: The bifurcation located at the ostium and real vessel size is not known.

- Summary: Renal artery angiography is the gold standard diagnostic test.

However, to make a correct diagnosis of ARAS, excellent visualization of renal vasculature should be performed. Aortography is now replacing to CT angiography. But in placing catheter to renal artery ostium, we still need aortography for the safe catheter manipulation. Selective angiography identifies the severity of stenosis with intra renal vasculature information. Confusion for the indication of renal artery stenting is mostly coming from poor angiographic image of renal artery stenosis.

\section{Iliac artery angiography}

Peripheral arterial disease at the level of iliac artery is well known for good indication of angioplasty. Currently, stenting for the treatment of iliac occlusive disease is the most effective modality and endovascular treatment of iliac artery disease should be considered as a first-line therapy for symptomatic PAD. The most commonly quoted classification of iliac lesions has been set forth by the TransAtlantic interSociety Consensus (TASC II) group with recommended treatment options (13). The type A and B lesions are treated preferentially by endovascular techniques and typed C and D lesions are more suited for surgical treatment. However, recent development of endovascular technique, even type $\mathrm{D}$ lesions sometimes is treated by endovascular procedure. To maximize the success of iliac artery stenting, good quality angiogram is needed. 


\subsection{Aortography for iliac artery disease}

artery. The image field at least $30 \mathrm{~cm}$ is needed. In Fig. Iliac $1,31 X 31 \mathrm{~cm}$ and $30 X 40 \mathrm{~cm}$ image field are shown (Fig. Iliac 1). In both angiogram show from terminal aorta to common femoral artery. However, in $31 \mathrm{~cm}$ image field, we are lifting a table to maximize image field ( Fig. Iliac 2). Typical long left iliac artery total occlusion is shown in Fig Iliac 2A. This image was taken by lifting table to obtained collateral vessels to common femoral artery (Fig. Iliac 2B). DSA image is considered to be the standard angiography for iliac artery disease, but pelvic vessels often times interfered by bowel movement and gas. In recent advanced digital angiography, similar image to subtracted angiogram can be obtained. In Fig. Iliac 3, subtracted (In Fig. Iliac 3A) and nonsubtracted (In Fig. Iliac 3B) are shown. Even nonsubtracted image is acceptable with bony landmark.
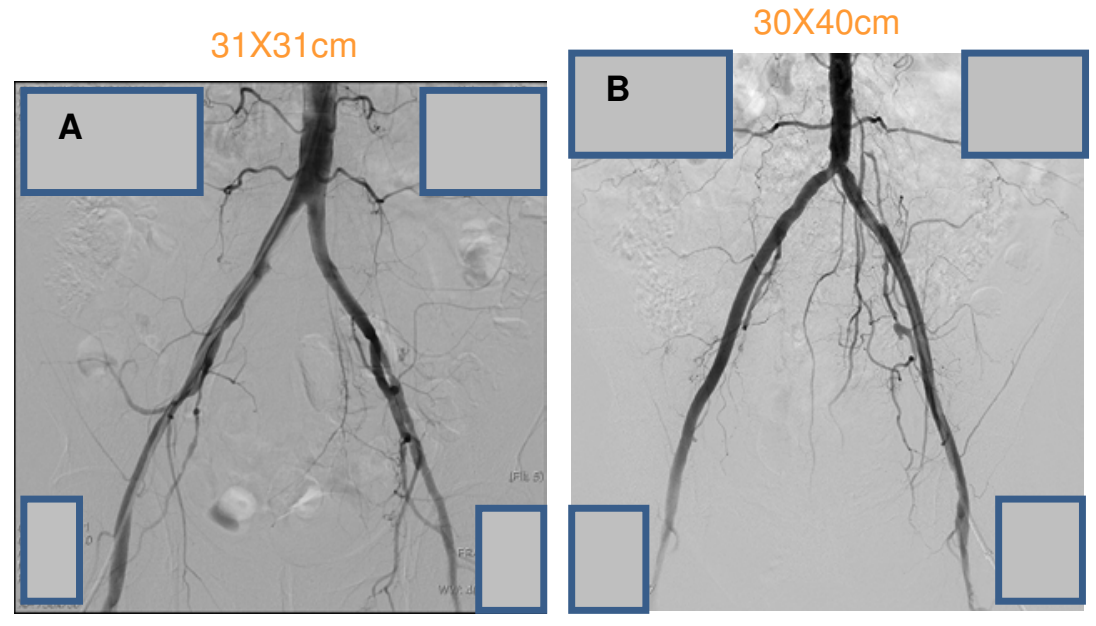

Fig. Iliac 1: Aortography for iliac artery

A: $31 \times 31 \mathrm{~cm}$ image,

B: 30X40cm image

Both angiogram could show from terminal aorta to common femoral artery.

\subsection{Image size}

To visualize aorto iliac disease, more than $30 \mathrm{~cm}$ image field is required. But in reality, image of $30 \mathrm{~cm}$ often times misses the complicated stenostic lesion. In Fig. Iliac $4 \mathrm{~A}$, there is a tight stenosis in left external iliac artery. In $20 \mathrm{~cm}$ image, ulcerated tight stenosis in left common iliac artery clearly visualized (Fig. Iliac 4B). To intervene the iliac artery disease, the information of vessels size and lesion length is needed. $30 \mathrm{~cm}$ image could confirm the disease of common femoral artery, but to obtain the precise lesion morphology, $20 \mathrm{~cm}$ image is better than $30 \mathrm{~cm}$ image.

\subsection{Basic 3 views for iliac artery}

The anteriorposterior (AP) pelvic angiogram is the basic angiogram. The oblique images should be obtained. If contrast load permits, basically we are taking three view of aortogram 

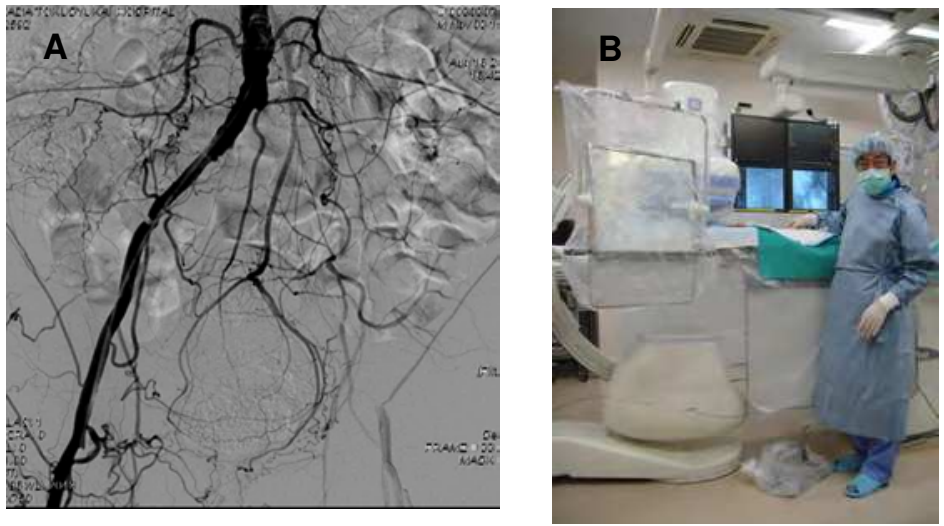

Fig. Iliac 2: Aortography by $31 \times 31 \mathrm{~cm}$ image field A: Aortography of $31 X 31 \mathrm{~cm}$ image. Left common femoral artery is visulaized through collateral.

B: Catheter table is lifted to maximize image field
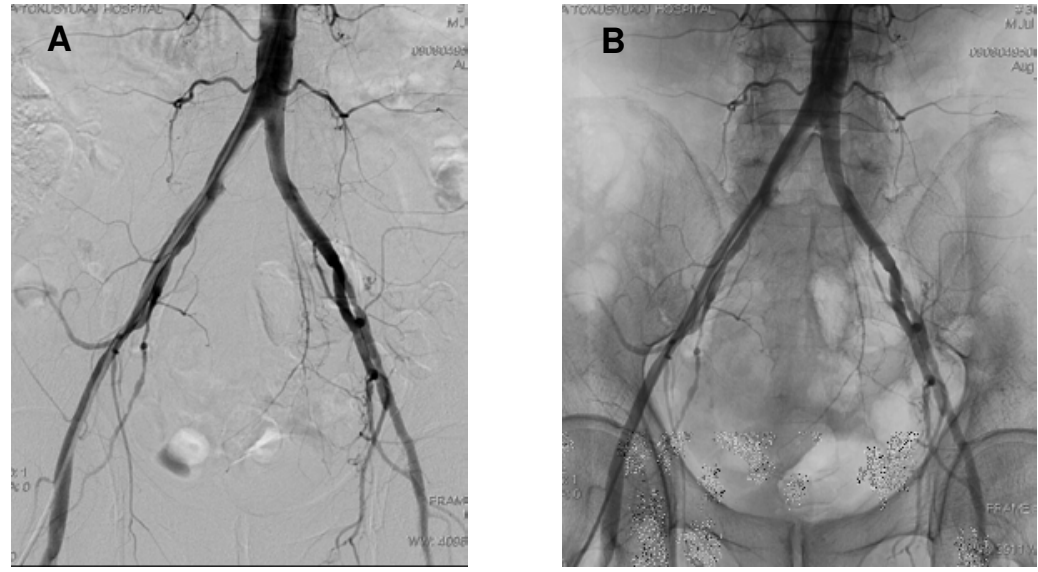

Fig. Iliac 3: Subtracted and non subtracted Aortography

A: Image of digital subtraction angiography

Image of digital subtraction angiography is considered to be the standard for the visualization of iliac artery disease

B: Non subtracted image

Pelvic vessels are interfered by bowel movement and bowel gas. Recent digital angiography could give the similar image to subtracted angiogram. 

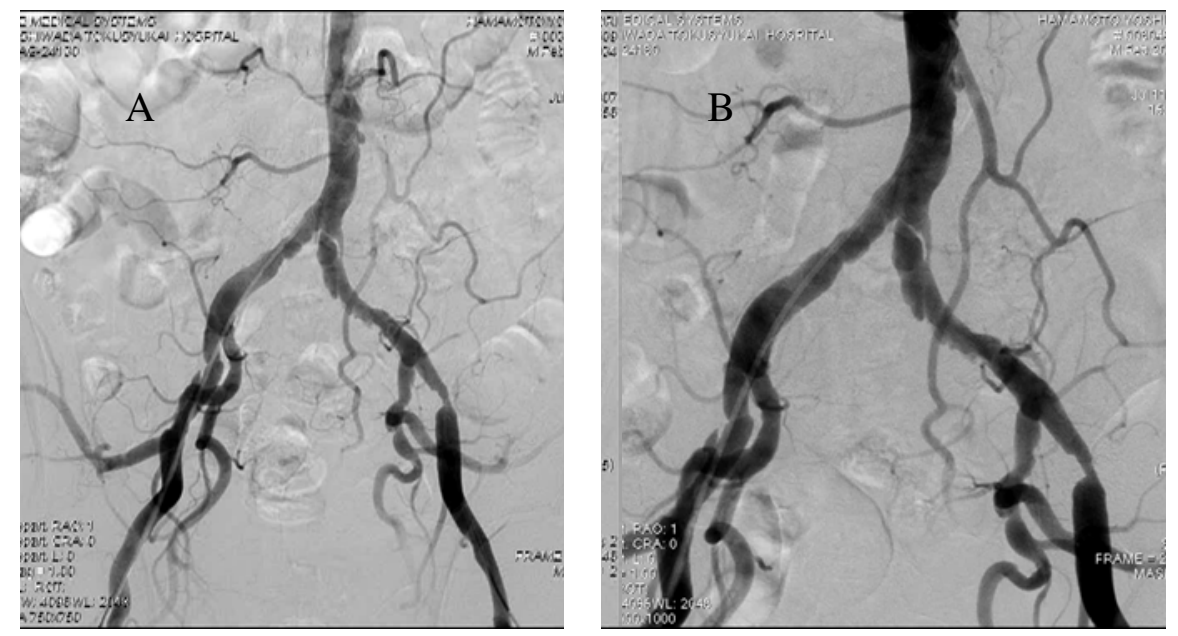

Fig. Iliac 4: Image size of Iliac artery disease

A: $31 \mathrm{~cm}$ of image

B: $20 \mathrm{~cm}$ of image

To visualize iliac artery disease, more than $30 \mathrm{~cm}$ image field is required. But for the purpose of interventional work, image of $30 \mathrm{~cm}$ often times misses the complicated stenotic lesion. There is a tight stenosis in left external iliac artery(A). In $20 \mathrm{~cm}$ image, ulcerated tight stenosis in left common iliac artery clearly visualized (B).

(Fig. Iliac 5). In a right anterior oblique view, the left iliac artery is best visualized and could separate bifurcation of internal iliac artery (Fig. Iliac 5A). Similarly, right iliac artery is taken by left anterior oblique (Fig. Iliac 5C) with the projection $30^{\circ}$. In complex disease anatomy, this approach is very important before intervention. In Fig. Iliac 6, severely diseased bilateral external iliac artery are shown in three views. In AP view, right external iliac artery over rap and left external iliac artery is not well visualized (Fig. Iliac 6B). The right internal anterior oblique view shows occlusion of left external iliac artery iliac artery (Fig. Iliac 6A). Left anterior view clearly delineate right external and internal iliac artery (Fig. Iliac 6C).

\subsection{Working image for intervention}

In iliac artery disease, primary iliac stenting is performed in most of the cases. To stent, exact lesion location must be visualized. In Fig. Iliac 7, left external iliac artery focal stenosis is shown (Fig. Iliac 7A). In DSA image, bony landmark is not seen and DSA image converted to non-subtracted image (Fig. Iliac 7B). By seeing femoral head, precise stenting was performed (Fig. Iliac 7C).

\subsection{Contrast injection from sheath}

Iliac artery stenting is becoming the routine procedure and angiography can be simplified. In ipsilateral retrograde femoral approach, sheath is placed and this sheath can be used 
RAO

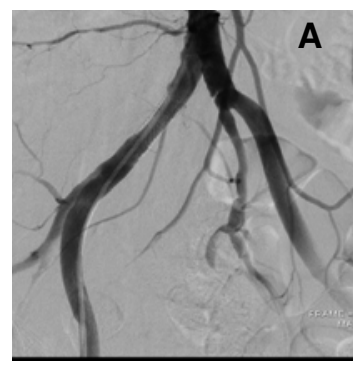

AP

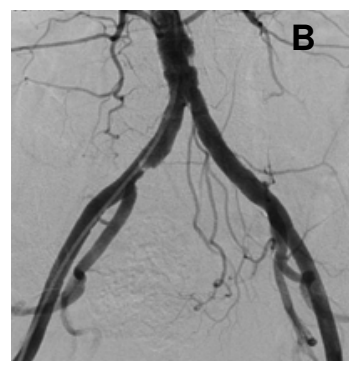

LAO

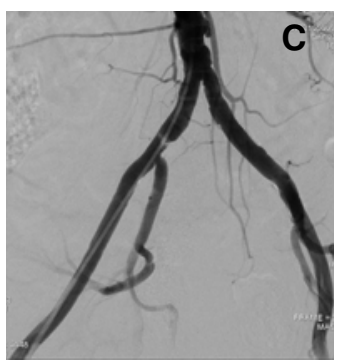

Fig. Iliac 5: Basic 3 views for iliac artery

A: $30^{\circ}$ right anterior oblique view

B: Anteriorposterior (AP) view

C: $30^{\circ}$ left anterior oblique view

In a right anterior oblique view, the left iliac artery is best visualized and separate bifurcation of internal iliac artery (A). In AP view, both external iliac artery are shortened(B) Similary the right iliac artery is taken by a left anterior oblique with the projection $30^{\circ}$ angle $(\mathrm{C})$.

RAO

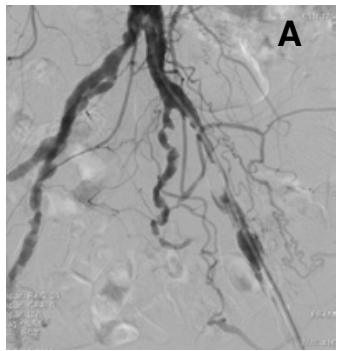

AP

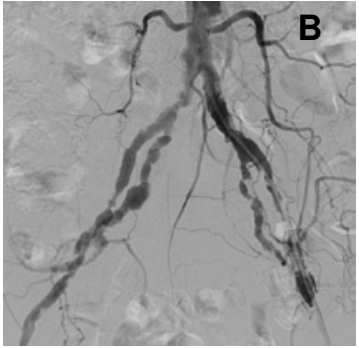

LAO

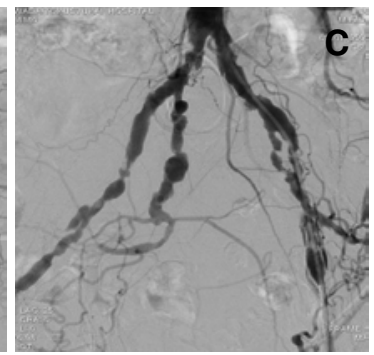

Fig. Iliac 6: Basic 3 views for iliac artery diease

A: $30^{\circ}$ right anterior oblique view

B: Anteriorposterior (AP) view

C: $30^{\circ}$ left anterior oblique view

The right internal anterior oblique view shows occlusion of left external iliac artery iliac artery (A). In AP view, right external iliac artery over rap with right internal iliac artery and left external iliac artery is not well visualized (B). The left anterior view clearly delineate right external and internal iliac artery and shows diffusely diseased right external iliac artery (C). 

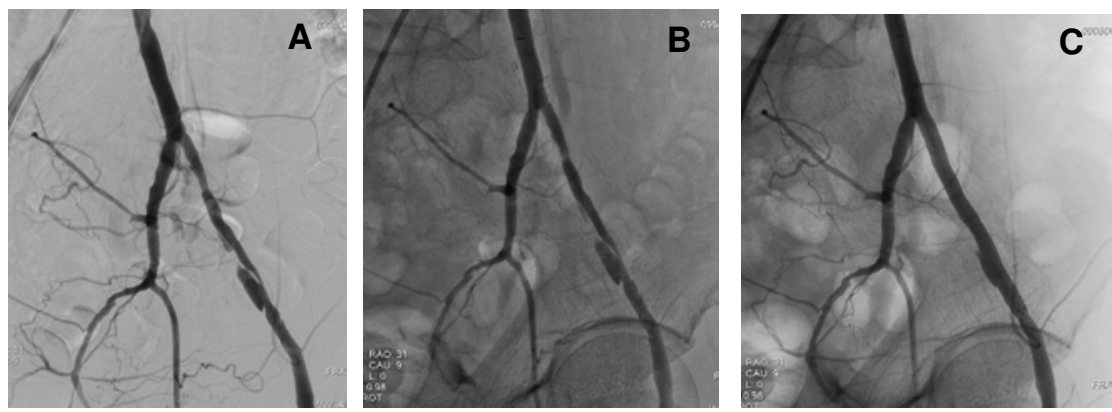

Fig. Iliac 7: Working image for intervention

A: Subtracted image

B: Non subtravted image

C: Post stenting

To stent, exact lesion location has to be visualized. Foca stenosis in left external iliac artery is shown by DSA image (A). Bony landmark is not seen and DSA image converted to nonsubtracted image (B). By seeing femoral head, precise stenting was performed (C).
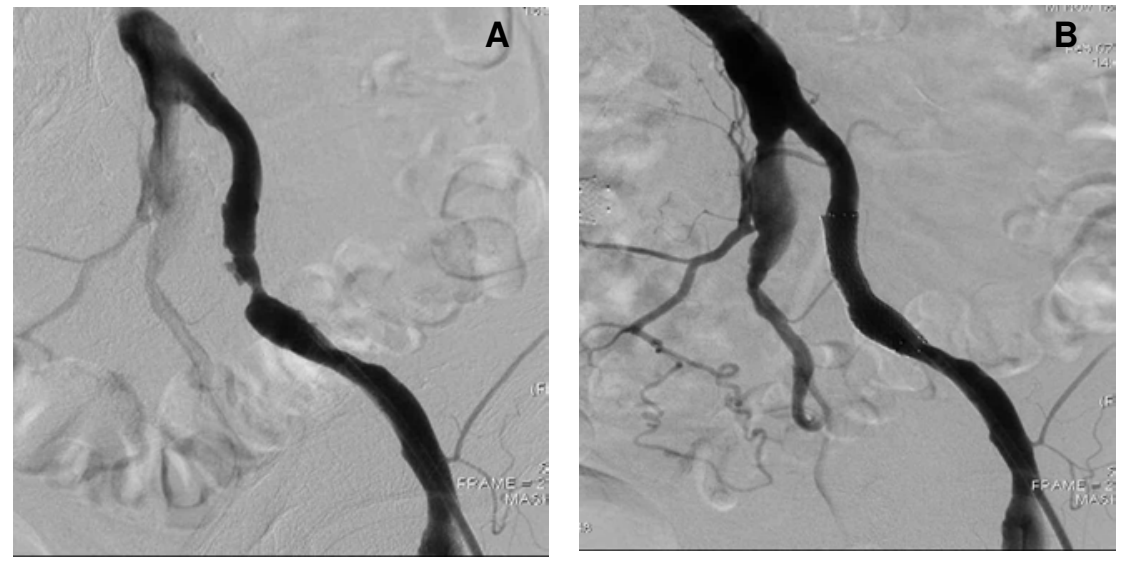

Fig. Iliac 8: Ipsilateral contrast injection from sheath

A: Angiogram by injecting dye through left femoral artery sheath

B: Post stenting angiogram

In ipsilateral retrograde femoral approach, sheath can be used to injection of dye. There is a focal stenosis in left external iliac artery (A). Angiogram was performed through this sheath and ballooning and stenting was performed. (B).

to injection of dye. There is a focal stenosis in left external iliac artery (Fig. Iliac 8A). Angiogram was performed through this sheath and ballooning and stenting was performed. (Fig. Iliac 8B). However, in retrograde approach, hand injection cannot visualize proximal iliac artery. Power injector should be employed and test injection is needed to check the tip of sheath position is in vessel lumen. 


\subsection{Summary}

Iliac artery stenting is recognized as an effective treatment and became the standard therapy for iliac artery disease. CTA and vascular echo can be utilized for diagnostic purpose and diagnostic angiography is less performed. But pelvic vessels are difficult to diagnose by echo. CTA can be misread by calcification. The angiogram is still the golden standard for final decision making of iliac artery stenting.

\section{Femoropopliteal artery angiography}

Patients with disease limited to superficial femoral artery (SFA) usually present with claudication. However, most of severe claudication shows multi level involvement. In SFA, popliteal and infrapopliteal artery disease could be a cause of critical limb. Interventional approach to SFA is much easier than infrapopliteal artery and precise assessment of femoropopliteal artery disease in very important to deal with severe claudication and critical limb.

\subsection{Proximal femoral artery}

proximal femoral artery disease. In AP view, profunda femoral override to SFA (Fig. SFA 1A). In ipsilateral oblique view, separation between SFA proximal and profund femoral are well seen (Fig. SFA 1B). This view is particular important for diseased proximal femoral artery. The SFA proximal stenosis is not delineated by anterior posterior (AP) view (Fig. SFA 2A). Left anterior oblique view shows SFA ostial stenosis and found that lesion length of proximal profunda artery is longer than AP view (Fig. SFA2B). This angled view is particular useful to guide the wire into SFA ostial occlusion. In Fig SFA 3 shows the typical SFA long occlusion originating from SFA ostium (Fig. SFA 3A). In AP view, SFA ostium is not identified (Fig. SFA 3B). In left anterior oblique view, SFA ostium occlusion is well visualized (Fig. SFA 3C).

\subsection{Bolus chase for limb artery angiography}

To see the entire limb artery, a bolus chasing angiography is very useful method. In some angiographic system, this angiography can be done by digital subtraction with small amount contrast. We usually give $4 \mathrm{cc} /$ second, total $16 \sim 18 \mathrm{cc}$ of contrast to visualize from femoral to tibial artery. In Fig SFA 4A is the bolus chase of right limb. Infrapopliteal arteries are not well seen, but this angiogram give the right limb is not severely diseased. In Fig. SFA 4B shows the two focal stenosis with three tibial vessels run-off. The typical SFA occlusion with well developed collateral via profunda femoral artery is well seen (Fig. SFA4C).

\subsection{Lesion length}

marking is placed in SFA. Tape measurement is fairly consistent with marker wire and this tape can be used to measure lesion length (Fig. SFA 5). By using this tape, lesion length can be measured. Short focal lesion was measured at $1 \mathrm{~cm}$ (Fig. SFA 6A). The lesion length of short CTO is measured at $6.5 \mathrm{~cm}$. In Fig. SFA $6 \mathrm{C}, 20 \mathrm{~cm}$ long CTO is shown. In long diffuse lesion, another way to measure lesion length is to employ balloon marker. In Fig. SFA 7A, long SFA lesion is shown. By using $10 \mathrm{~cm}$ balloon marker (Fig. SFA 7B), lesion was calculated about $20 \mathrm{~cm}$ and two $6 \mathrm{mmX} 100 \mathrm{~mm}$ stent were implanted (Fig. SFA 7C). 

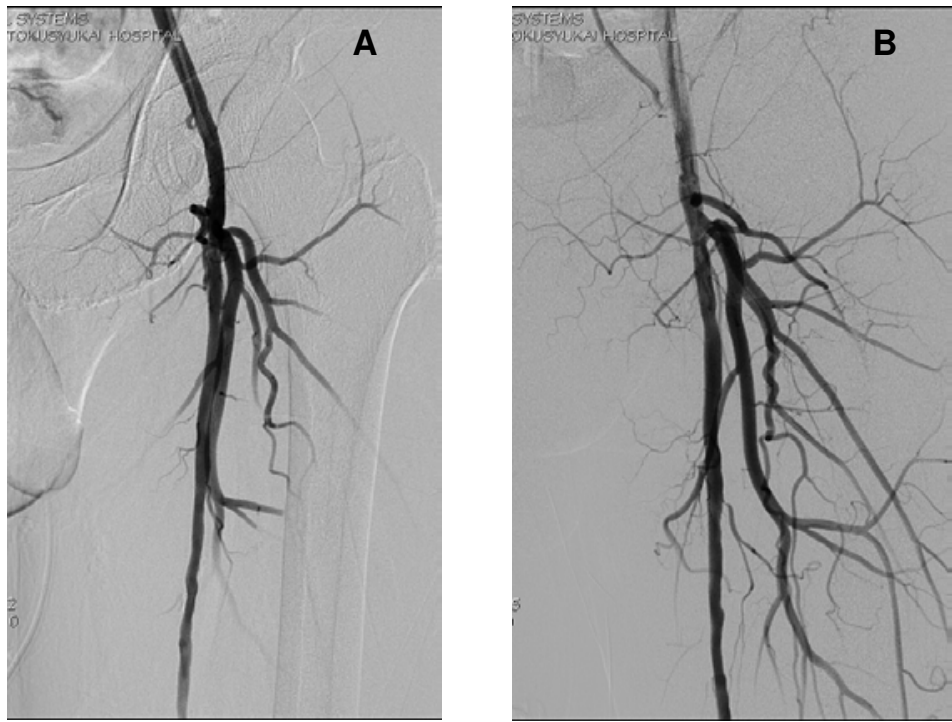

Fig. SFA 1: Anteroposterior view and left anterior oblique view for proximal left femoral artery

A: AP view shows that profunda femoral override to SFA

B: In left anterior oblique view, separation between SFA proximal and prfund femoal are well seen
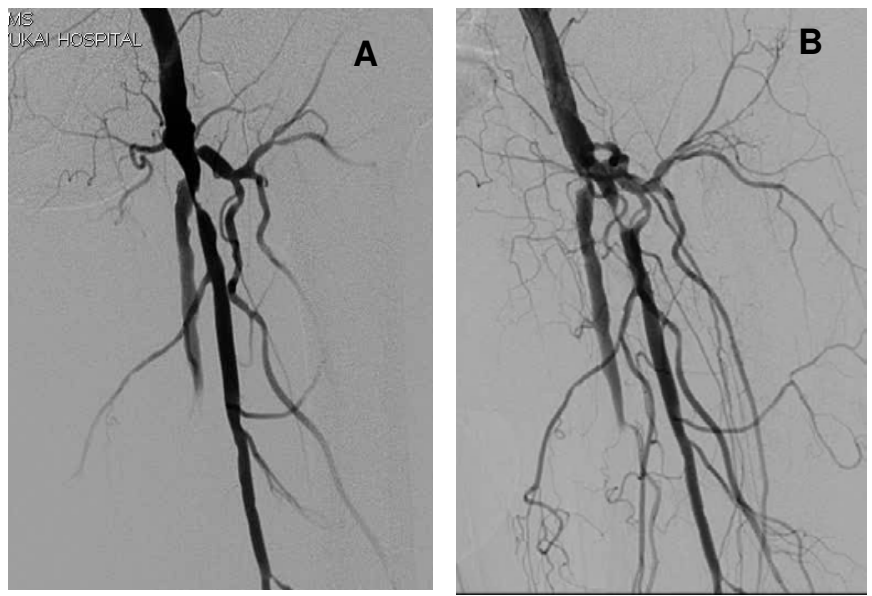

Fig. SFA 2: Anteroposterior view and left anterior oblique view for proximal left femoral diseased artery A: The SFA proximal stenosis is not delineated by anterior posterior (AP) view B: Left anterior oblique view shows SFA ostial stenosis and lesion length of proximal profunda artery is longer than AP view 

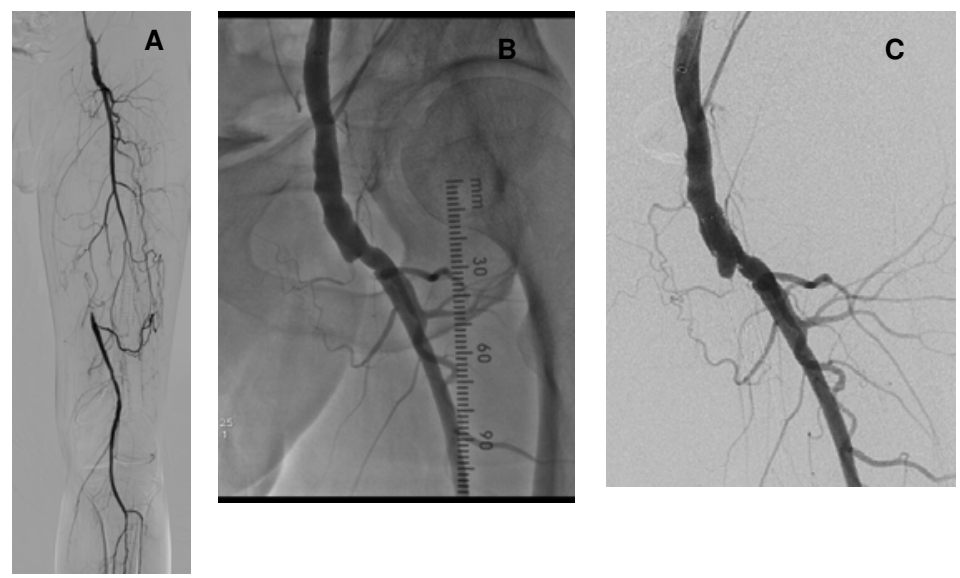

Fig. SFA 3: Anteroposterior view and left anterior oblique view for proximal left femoral artery occlusion A: Typical SFA long occlusion originating from ostium B: In AP view, SFA ostium is not clearly seen

C: In left anterior oblique view, the stump of SFA ostium occlusion is well visualized
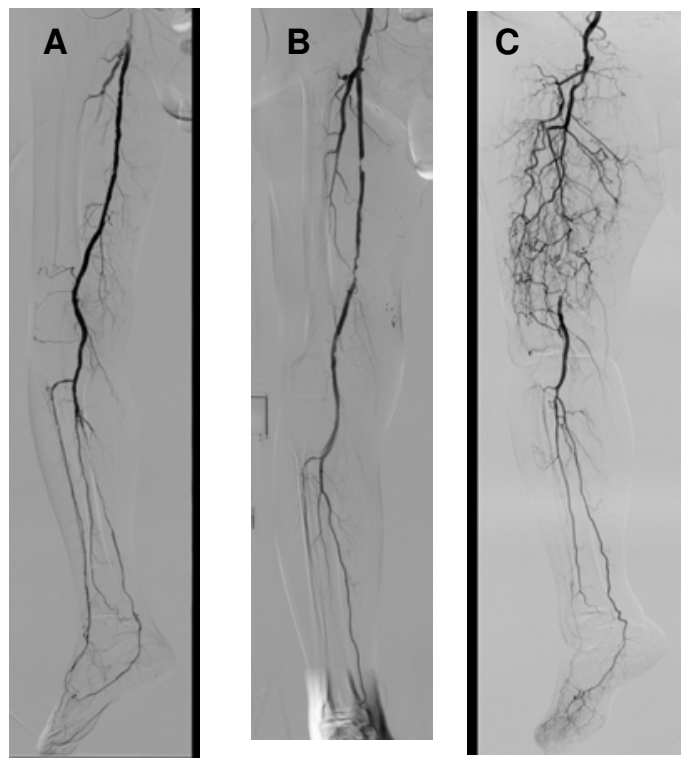

Fig. SFA 4: Bolus chase for limb vessel angiography

To see the entire limb artery, bolus chasing is very useful method. This angiogram can be obtained by digital subtraction with small amount dye.

A: Bolus chase of right limb. Infrapopliteal arteries are not well seen, but this angiogram shows the right lower limb is not severely diseased.

B: Two focal stenosis with three vessels run-off is shown

C: Typical SFA occlusion with well developed collateral via profunda femoral artery is well seen 


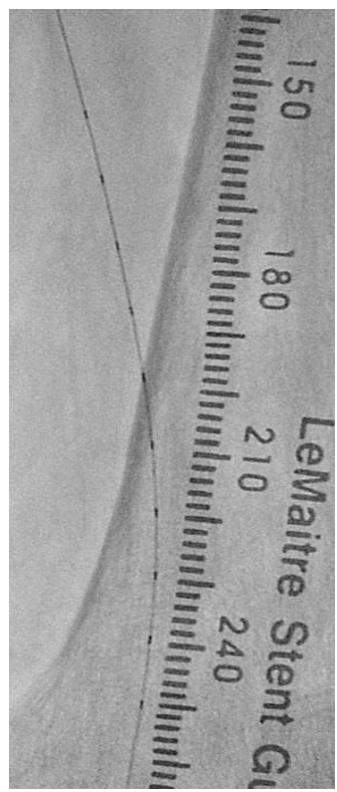

Fig. SFA 5: Marker wire and measure tape

To measure lesion length, maker tape is attached to frontal leg muscle. This tape is validated with marker wire placed in SFA. This tape can be used to measure lesion length.
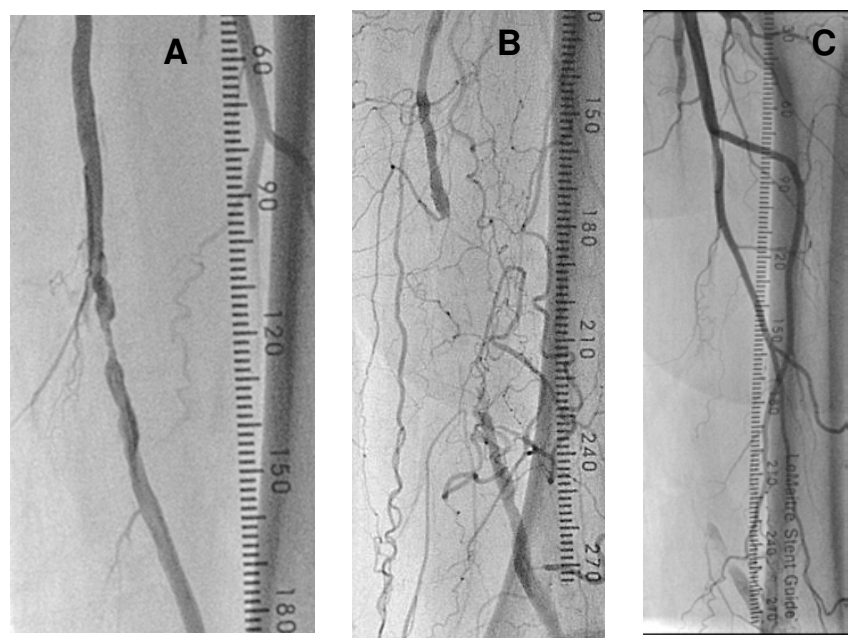

Fig. SFA 6: Lesion length of SFA disease

A: Focal lesion was measured at $1 \mathrm{~cm}$

B: Short CTO was measured at $6.5 \mathrm{~cm}$.

C: SFA long CTO was measured at $20 \mathrm{~cm}$ 

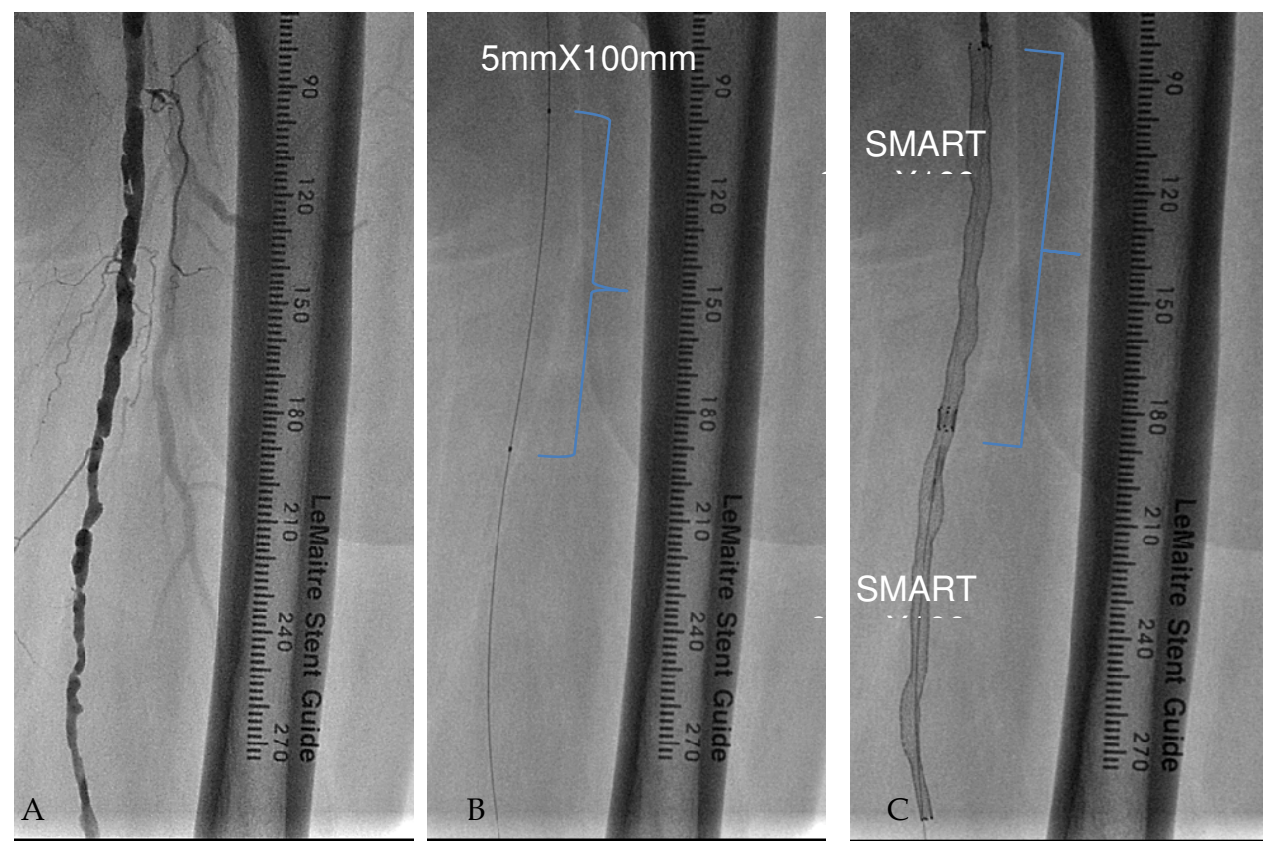

Fig. SFA 7: Lesion length measured by balloon marker A: SFA long diffuse lesion

B: Measring lesion length by using $10 \mathrm{~cm}$ balloon marker

C: Lesion was calculated about $20 \mathrm{~cm}$ and two $6 \mathrm{mmX} 100 \mathrm{~mm}$ stent were implanted

\subsection{TASC II Classification(13)}

Before intervening the lesion, lesion length and morphology can be classified according to TASC II guideline (13). It will give the standard indication of interventional treatment or bypass surgery. Fig. SFA 8 A shows a single focal lesion less than $10 \mathrm{~cm}$ in length and did not involve the origins of SFA. This lesion is classified into type A (Fig. SFA8A). Fig. SFA 8B is a single CTO lesion less than $15 \mathrm{~cm}$ and considered to be type B (Fig. SFA 8B). Fig. SFA 8C shows multiple stenosis more than $15 \mathrm{~cm}$ lesion length and a typical example of type C (Fig. SFA 8C). Fig. SFA 8D shows the long CTO and lesion length is more than $20 \mathrm{~cm}$ (Fig.SFA 8D). This is the typical type $\mathrm{D}$ and stenting for this kind of lesion could be a high chance of restenosis.

\subsection{Ballooning or stenting}

In iliac artery disease, primary stenting is firmly established. In femoropopliteal artery disease, it is still controversial about primary stenting. In short lesion, the initial approach is still balloon angioplasty (Fig. SFA 9A, B). Angiogram of post balloon angioplasty shows suboptimal result (Fig. SFA 9C). But in reality, most of the lesion needs to be stented. Fig. SFA $10 \mathrm{~A}$ shows the typical $5 \mathrm{~cm}$ stenostic lesion and balloon angioplasty was performed (Fig. SFA10B). Balloon dilatation resulted in dissection and bail out stenting was performed. Nitionol stenting could seal dissection with no residual stenosis (Fig. SFA 10C). 

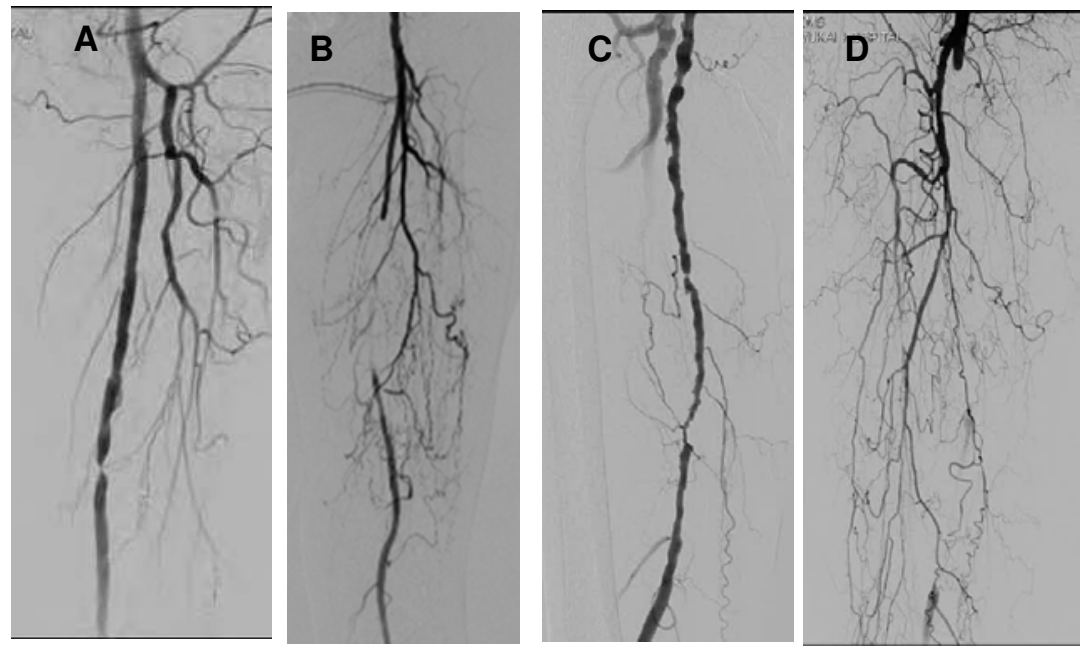

Fig. SFA 8: Lesion classification based on TASC 11 guideline (13)

TASC II guideline gives the standard indication of interventional treatment or bypass surgery.

A: TASC A is a single focal lesion less than $10 \mathrm{~cm}$ in length

$B$ : TASC B is a single CTO lesion less than $15 \mathrm{~cm}$

C: TASC C is multiple stenosis more than $15 \mathrm{~cm}$ lesion length

$\mathrm{D}$ : TASC D is a long CTO and lesion length is more than $20 \mathrm{~cm}$
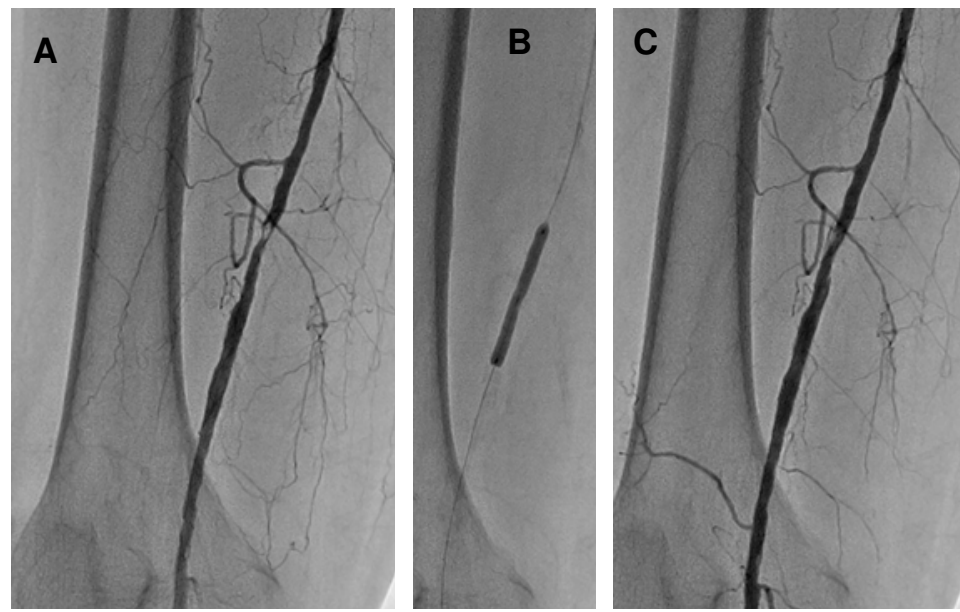

Fig. SFA 9: Balloon angioplasty

In short lesion, initial approach is the balloon angioplasty

A: A sort focal lesion in distal SFA

B: Balloon angioplasty was performed $5 \times 40 \mathrm{~mm}$ balloon at 8 ATM

C: Post balloon angioplasty showed a residual less than $50 \%$ stenosis at ballooning site 
Pre

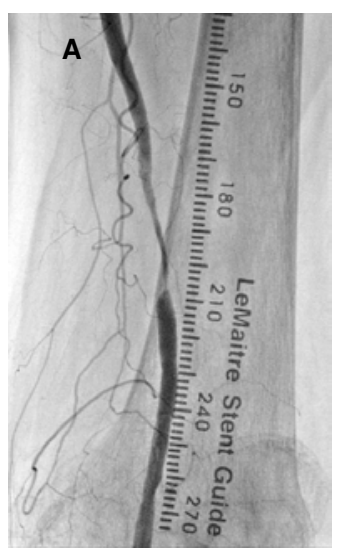

Post ballooning

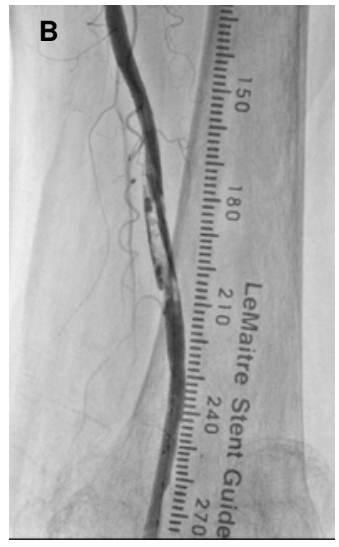

Post stenting

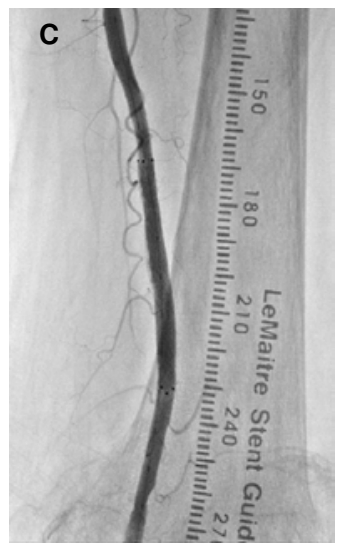

Fig. SFA 10: Bail out stenting post balloon angioplasty

A: $5 \mathrm{~cm}$ stenostic lesion

B: Balloon angioplasty was performed and resulted in dissection

C: Nitinol stenting for bail out purpose succeeded to seal the dissection

\subsection{Stent fracture}

Stent fracture in SFA is a growing concern (14). In most of the cases, stent fracture are not related to restenosis. Wall stent was implanted at 8 years ago (Fig. 10A). The stent showed complete traverse liner separation without stent displacement. This is called type 3 stent fracture. But there is a stent fracture related to restenosis. In Fig. SFA 11A, there is a focal restenosis at SMART stent implanted site. In plain view, multiple separation of strut is seen (Fig. SFFA 11B). Magnified view shows, multiple stent strut were separated (Fig. SFA 11C). This stent fracture could be a cause of restenosis.

\subsection{Stent restenosis}

In recent years, studies have demonstrated the superiority of stents over balloon angioplasty as far as primary patency is concerned (15). In some center, SFA Nitinol stent is empoloyed as primary use. However, stent restenosis is still the big issue for primary stenting. SFA long CTO originating from SFA ostium was shown in Fig. SFA 12A. Successful recanalization was obtained with stenting (Fig. SFA 12B). Angiogram at 6 months showed stent restenosis with new stenosis in profund femoral artery (Fig. SFA 12C). This restenosis is clearly seen in non subtracted angiogram (Fig. SFA12D). This suggest stent affecting to other vessel as the chronic cell proliferation.

\subsection{Summary}

SFA is the longest vessel and is difficult to visualize entire vessel. In proximal part of SFA should be taken by ipsilateral view. To evaluate lesion severity, lesion length is the important factor for endovascular approach. The precise lesion length measurement must be made. Stent restenosis after endovascular treatment of SFA obstructions is still the big concern. 

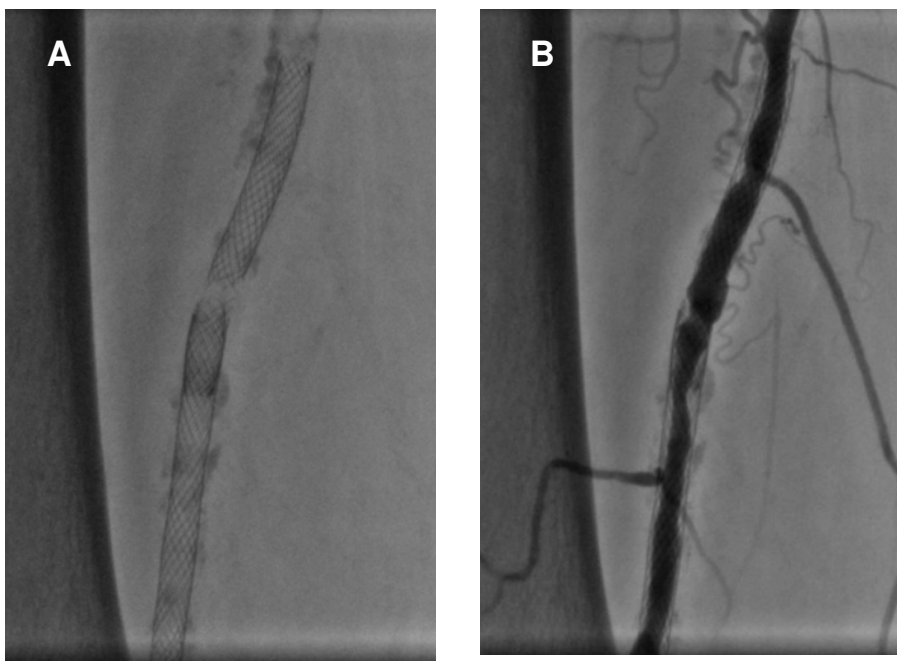

Fig. SFA 11: Stent fracture

A: Wall stent was implanted at 8 years ago. The stent showed complete traverse liner separation without stent displacement. This is called type 3 stent dissection.

B: There is no restenosis at fractured site
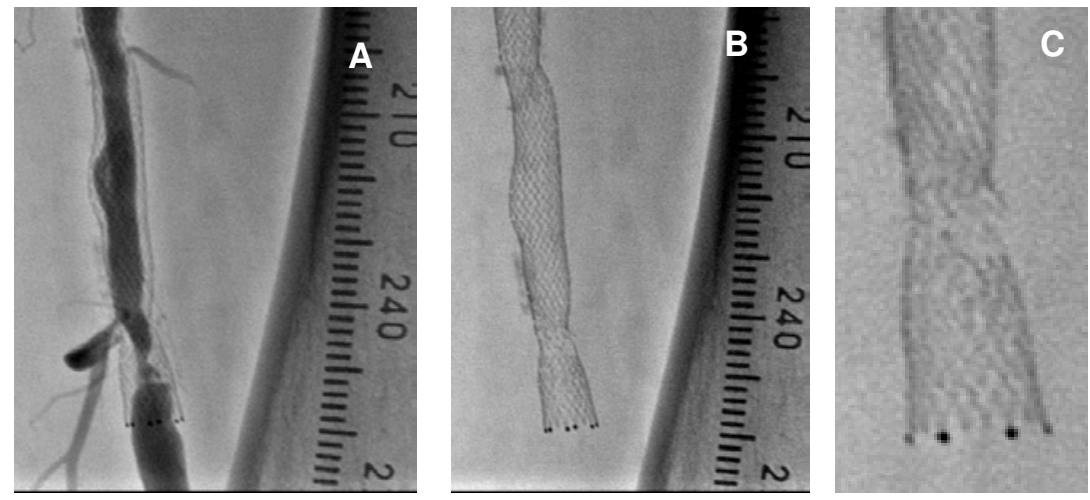

Fig. SFA 12: Stent fracture with restenosis

A: There is a focal restenosis at SMART stent implanted site.

B: In plain view, multiple separation of strut are seen

C: More magnified view shows, multiple stent strut were separated

\section{Below the knee angiography}

Infrapopliteal atherosclerotic lesions are common in critical limb ischemia and to assess these lesions, a meticulous angiographic technique should be taken. In Below the knee (BTK) angiogram, 4 BTK arterial segments (tibioperoneal trunk, anterior tibial, posterior tibial arteries and peroneal artery) must be separated and identified. And also, continuation 

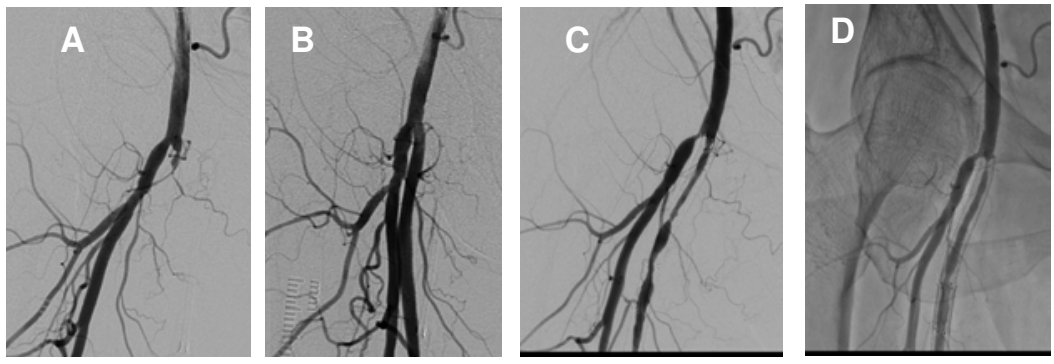

Figure SFA 13: Stent restenosis

A: SFA occlusion originating from ostium

B: Succeful recanalization was obtained with Nitinol stenting

C: At 6 months, angiography showed SFA stent restenosis with a new stenosis in profund femoral artery.

D: This restenosis is clearly seen in non subtracted angiogram

from anterotibial artery to dorsal pedis and posetotibial to planter artery have to be delineated. BTK arteries are most complicated vessels and difficult to identify each three vessels. Meticulous angiographic approach should be taken to visualize three tibila arteries.

\subsection{Basic angiography for BTK}

Femoropopliteal artery angiogram must be taken to rule out inflow disease (Fig. BTK 1A). After confirming no inflow disease is present, BTK angiography is performed through the catheter located at distal popliteal artery (Fig. BTK 1B). This BTK angiogram is to show three tibial arteries and antero tibial artery is occluded. To locate exact lesion location, we take 4 views. These are two right anterior oblique view (RAO) (Fig. BTK 1C, 1D)and two left anterior oblique view (LAO) taken (Fig. BTK 1E, 1F). In upper RAO, proximal left anterotibial artery is well seen (Fig. BTK 1C). In lower RAO view, distal three tibial arteries are well seen (Fig. BTK 1D). In this case, distal anterotibial artery is occluded. In upper LAO, three tibial arteries are separated (Fig. BTK 1E). In lower LAO view, anterotibial and peroneal artery overrap and could not separate these two vessels (Fig. BTK 1F).

\subsection{Anatomical variation of infrapopliteal arteries}

Branching variations of the popliteal artery are not uncommon. A practical triad classification of the anatomical variation in the branching pattern is reported (17). Type 1 indicates a normal level of popliteal arterial branching, including the usual pattern (Fig. BTK 2A) and trifurcation (Fig. BTK 2B). Type 2 indicates a high division of popliteal artery branching. In Fig. BTK 2C, anterotibial artery arises at the knee joint. Type 3 indicates hypoplastic or branching with an altered distal supply, including a hypoplastic posterotibial (Fig. BTK 2D) and a hypoplastic antrotibial (Fig. BTK 2E) .There are more other variation, but we have to keep in mind variant tibial arteries are not uncommon.

\subsection{Numbers of patent tibial arteries}

After completing initial angiogram, we have to evaluate how many tibial arteries are patent. In normal BTK arteries, three vessels are patent (Fig. BTK 3A.) In Fig. BTK 3B, peroneal 

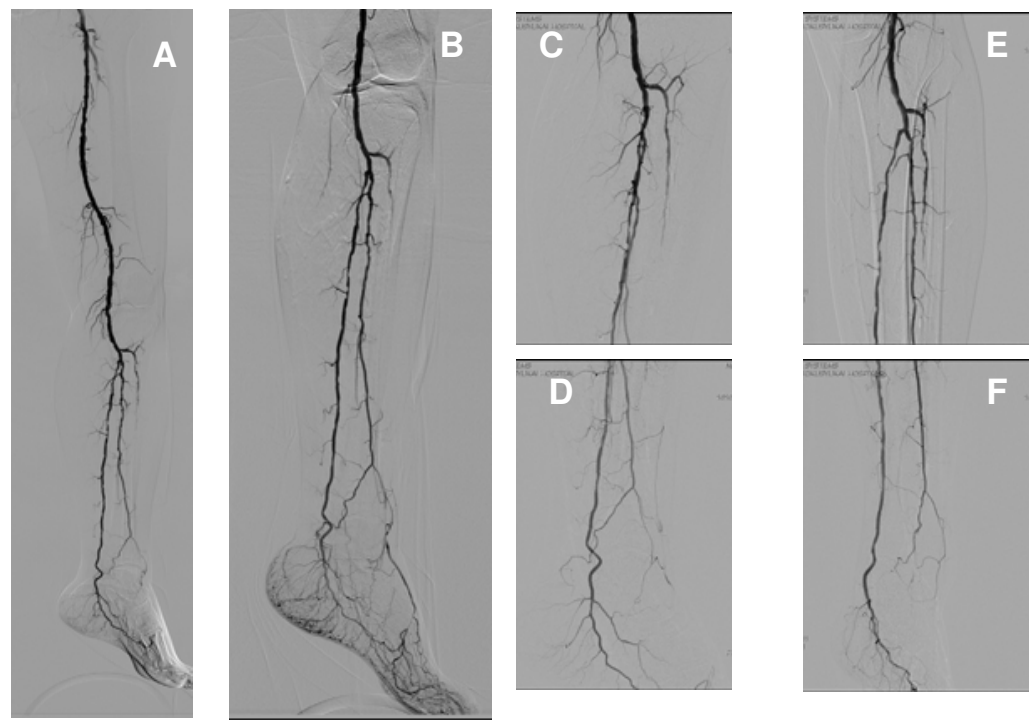

Figure BTK 1: Basic below the knee angiography

A: Ipsilateral left femoropopliteal to infrapoplital artery angiogram. This angiogram could rule out the inflow disease.

B: BTK angiography shows the entire three tibial arteries.

C: Upper right anterio oblique view.

Proximal left anterotibial artery is well seen. But posterotibial and peroneal artery are over rapped.

D: Lower right anterio oblique view. In this view, distal 3 tibial arteies are separated.

E: Upper left anterior oblique view.

Proximal three tibial arteries are well separated.

F: Lower left anterior oblique view.

Distal Posterotibial artery is well seen.

artery is not seen and considered two vessels run off. In Fig. BTK 3C, peroneal and posterotibial artery are patent and means two vessel runoff., In Fig. BTK 3D, only personal artery is patent and shows the one vessel run off.

\subsection{Single vessel run off of peroneal artery}

In Fig. BTK 4, both cases are single vessel disease with peroneal artery patent. These 2 cases, one peroneal artery is patent, but have active ulcer. It means that peroneal artery is not good enough for supplying foot arteries.(Fig. BTK 4). Peroneal artery terminates at the ankle and gives collateral to planter artery (Fig. BTK 4A). In Fig. BTK 4B, peroneal artery gives the collateral to dorsal pedis. This means to revascularize either anterotibial or posteriotibial artery must be recanalaized for the successful angioplasty.

\subsection{BTK angiogram of pre and post balloon angioplasty}

Before intervening BTK vessels, target vessel have to be clearly identified. In Fig. BTK 5, patient is a 65 year old man with diabetes and presented with right $4^{\text {th }}$ and $5^{\text {th }}$ toe ulcer. Pre 

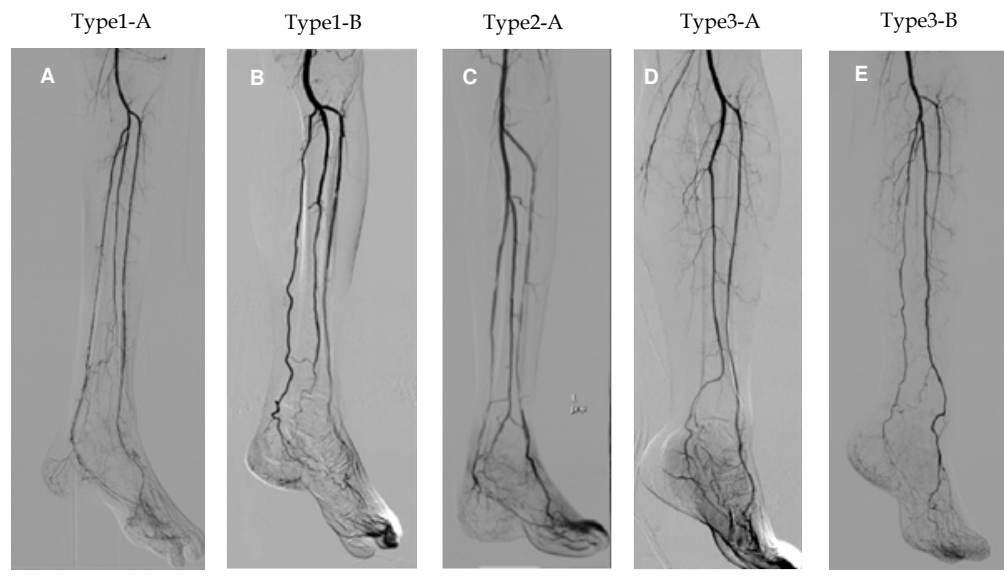

Fig. BTK 2: Anatomical variation of tibial arteries

A: Type 1-A

A normal level of arterial branching and most common pattern. The first brach is antrotibial artery and peroneal trunk separate to peroneal and posterotibial artery.

B: Type 1-B

A normal level of arterial branching. The three tibial arteries show trifurcation.

C: Type 2-A

A high division of popliteal artery branching and antrotibial artery arises at the knee joint.

D: Type3-A

Hypoplastic posterotibial artery and peroneal artery is giving a distal supply.

E: Type 3-B

Hypoplastic anterotibial artery and peroneal artery is giving a distal supply.

D: One vessel, peroneal arterry is patent
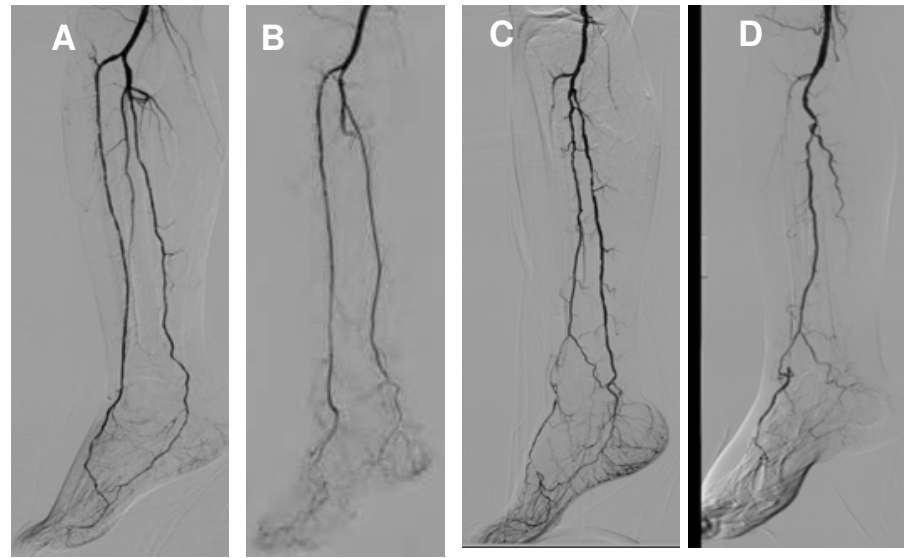

Fig. BTK 3: Number of patent tibial arteries

A: Three vessels are patent

B: Two vessels are with occluded peroneal artery

C: Two vessels are with occluded anterotibial artery 

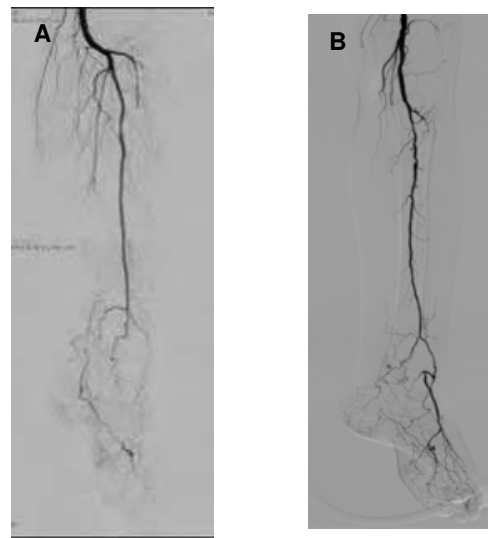

Fig. BTK 4: Single vesses runoff of peroneal artery in patients with critical limb A: Peroneal artery terminates at the ankle and gives collateral to planter artery B: Peroneal artery terminates at the ankle and gives collateral to dorsal pedis. In these two cases, single vessel are patent but ulcer does not heal. Peroneal artery is not giving sufficient flow distal to ankle.
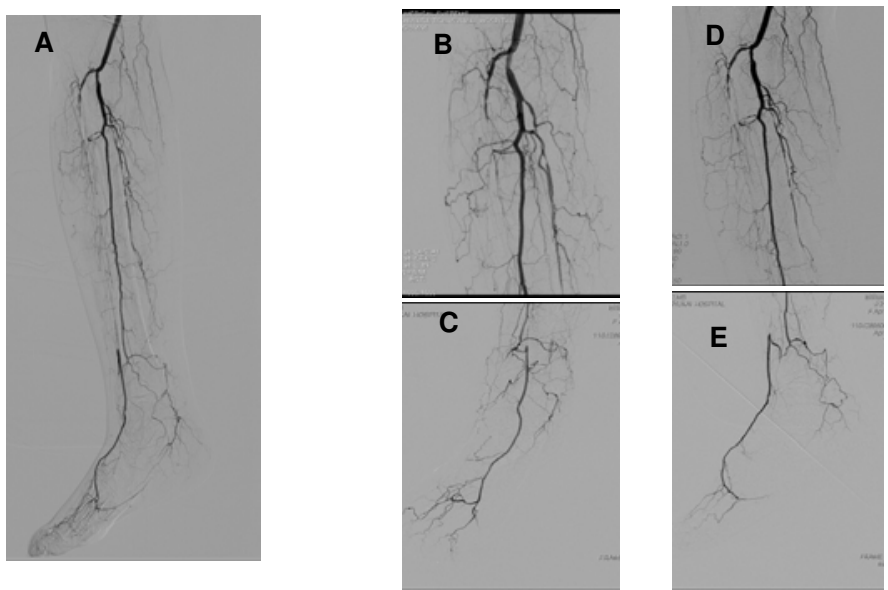

Fig. BTK 5: Pre interventional BTK angiography for critical limb ischemia A 65 year old man with diabetes and presented with right 4 th and 5 th toe ulcer. A: Bolus chase Right anterotibial and posterotibial arteries were occluded and peroneal artery is patent B: The upper RAO view

Three tibial arteries and right antrotibial artery was occluded at proximal portion $\mathrm{C}$ : The lower RAO view

Right dorsal pedis was patent; D: The upper LAO view - Only peroneal artery is patent $\mathrm{E}$ : The lower LAO view - Right antrotibial artery was occluded at the ankle and dorsal pedis artery is getting collateral through peroneal artery. Right planter artery is not well seen 
intervention angiography showed right anterotibial and posterotibial arteries were occluded (Fig. BTK 5A). Two right anterior oblique view (RAO) (Fig. BTK 5B, C) and two left anterior oblique (LAO) (Fig. BTK 5D, E) views were taken. The upper RAO showed three tibial arteries and right antrotibial artery was occluded at proximal portion (Fig. BTK 5B). The lower RAO view showed right dorsal pedis was patent (Fig. BTK 5C). The upper LAO view showed only peroneal is patent $t$ (Fig. BTK 5D). The lower LAO view revealed right antrotibial artery was occluded at the ankle and right planter artery is not well seen (Fig. BTK 5E). Based on these angiograms, right antrotibial artery occlusion was targeted. By using $2.5 \mathrm{~mm} X 10 \mathrm{~cm}$ over the wire balloon system, occluded anteoritibial artery was recanalaized (Fig. BTK 6). Post angioplasty angiogram was shown in Fig. BTK 7. Post intervention angiography showed right anterotibial was successfully recanalaized.and (Fig. BTK 7A). Two right anterior oblique view(RAO) (Fig. BTK 7B,C) and two left anterior oblique(LAO) (Fig. BTK 7D,E) views were taken to confirm the recanalized right antrotibial artery. The upper RAO showed three tibial arteries and right antrotibial artery was recanalized(Fig. BTK 5B). The lower RAO view showed peroneal override with anterotibial artery and could not separate two vessels(Fig. BTK 7C). The upper LAO view showed opened antrotibial artery(Fig. BTK 7D). The lower LAO view showed continuity between antrotibial to dorsal pedis was established (Fig. BTK 7E).
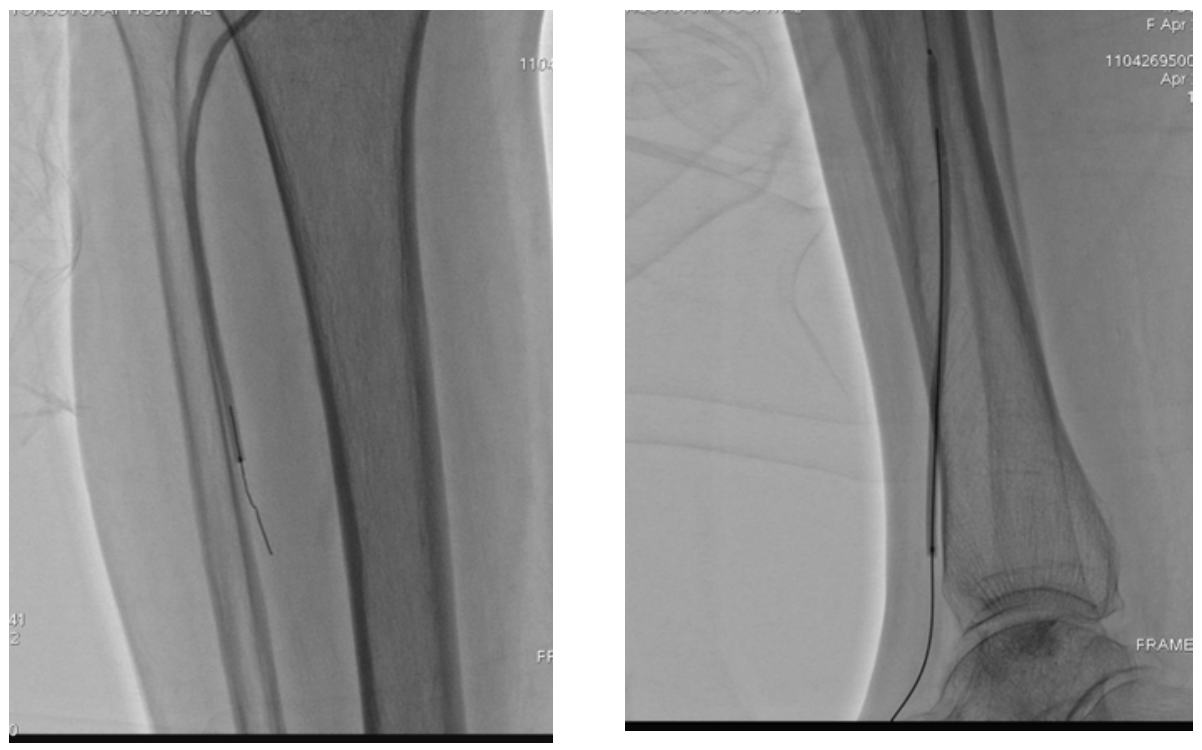

Fig. BTK 6: Balloon angioplasty to occluded anterotibial artery Based on these angiograms, right antrotibial artery occlusion was targeted.

A: By using $2.5 \mathrm{mmX} 10 \mathrm{~cm}$ over the wire balloon system, the occluded proximal anteoritibila artery was recanalaized

B: The wire advanced to dorsal pedis and succeeded to recanalization 

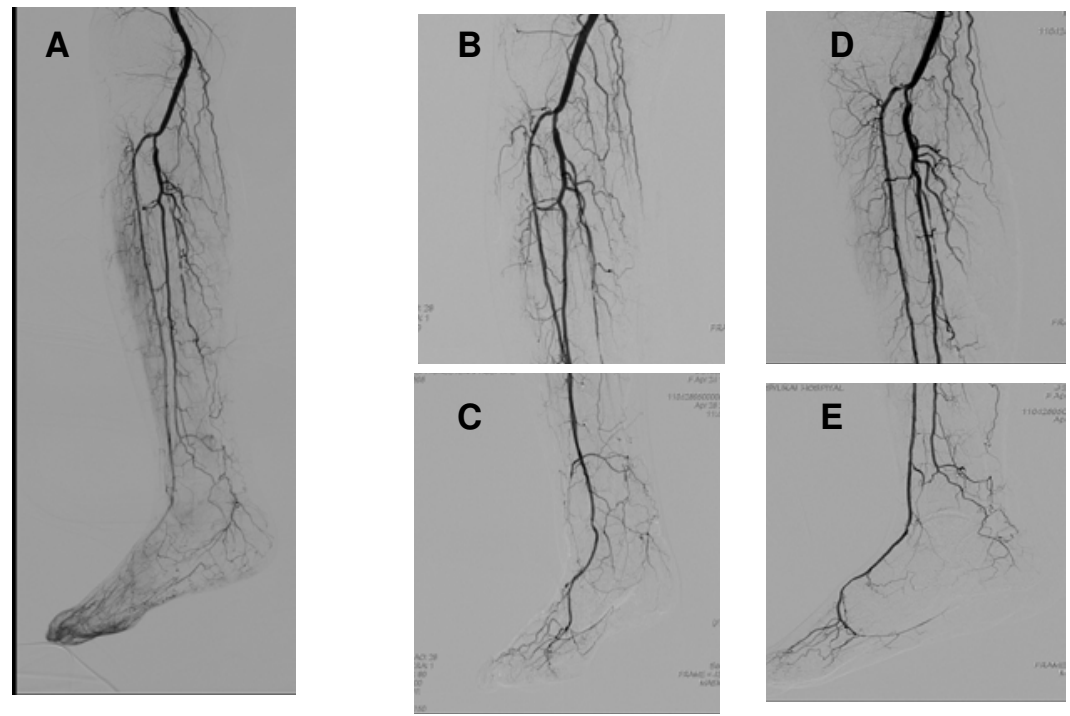

Fig. BTK 7: Post interventional BTK angiography for critical limb ischemia

A: Bolus chase

Right anterotibial artery was recanalized and straight line to dorsal pedis artery was established.

B: The upper RAO view

Right anterotibial artery was patent but midportion of anterotibial artery overrides with peroneal artery.

C: The lower RAO view

The continutity between anterotibial and dorsal pedis artery is confirmed.

D: The upper LAO view

Recanalized right anterotibial artey is well seen

E: The lower LAO view

Right anterotibial to dorsal pedis artey is shown.

\subsection{Foot artery angiogram}

In dealing with critical limb ischemia, precise knowledge of foot artery anatomy is needed. However, severe ischemic limb, foot artery angiogram is hard to obtain by the patient's leg movement. To minimize this problem, injection of small amount of contrast with inflow revascularization is mandatory. In Fig. BTK 8, typical ischemic foot artery is shown. Basically two views are good enough. One is antero-posterior view and the other is lateral view. In lateral view, complete occlusion of poterotibial artery is well seen and dorsal pedis show the short occlusion (Fig. BTK 8A). In anterior view, plantaris medial and lateralis are well separated (Fig. BTK 8B). And in anterior view, dorsalis pedis is well seen and suited for Retrograde dorsal pedis puncture (Fig. BTK 8B).

\subsection{Summary}

The effectiveness of BTK angioplasty for revascularization is well known (18). However, BTK angiography is one of the most difficult vessel for angiogram. Tibial arteries are small 

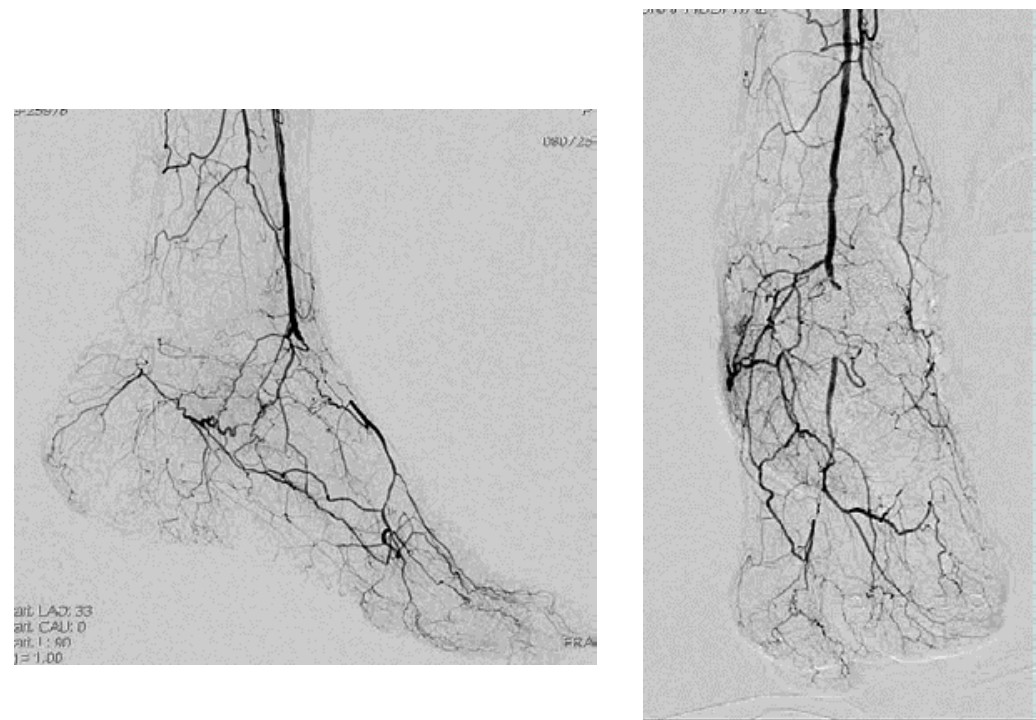

Fig. BTK 8: Foot artery angiogram:

A: Lateral view of left foot

Complete occlusion of poterotibial artery is well seen and dorsal pedis show the short occlusion

B: Anteroposterior view

Plantaris medial and lateralis artery are well separated. In anterior view, occluded dorsalis pedis artery is well seen

sized vessels with complicated anatomy. The amount of dye for injection is limited by patient's leg movement. However, angiogram is the only way to get the correct information BTK. The meticulous approach for BTK angiography should be taken.

\section{Reference}

[1] Wakhloo AK, Lieber BB, Seong J, Sadasivan C, Gounis MJ, Miskolczi L, Sandhu JS.Hemodynamics of carotid artery atherosclerotic occlusive disease. J Vasc Interv Radiol. 2004 ;15(1 Pt 2):S111-21.

[2] Sacco RL , Adams R, Albers G, et al; American Heart Association/American Stroke Association Council on Stroke; Council on Cardiovascular Radiology and Intervention; American Academy of Neurology. Guideline for prevention of stroke in patients with ischemic stroke or transient ischemic attack: a statement for healthcare professionals from the American Heart Association/American Stroke Association Council onStroke: co-sponsored by the Council on Cardiovascular Radiology and Intervention: the American Academy of Neurology affirms the value of this guideline. Circulation. 2006; 113:e409-e449. 
[3] Nedeltchev K, Pattynama PM, Biaminoo G, Diehm N, Jaff MR, Hopkins LN, Ramee S, van Sambeek M, Talen A, Vermassen F, Cremonesi A; DEFINE Group. Standardized definitions and clinical endpoints in carotid artery and supraaortic trunk revascularization trials. Catheter Cardiovasc Interv. 2010 Sep 1;76(3):333-44.

[4] Saba L, Mallarini G. MDCTA of carotid plaque degree of stenosis: evaluation of interobserver agreement. AJR Am J Roentgenol. 2008 Jan;190(1):W41-6.

[5] North American Symptomatic Carotid Endarterectomy Trial Collaborators. Beneficial effect of carotid endarterectomy in symptomatic patients with high-grade stenosis. N Engl J Med 1991;325:445-53.

[6] Peeters P, Verbist J, Deloose K, Bosiers M. Endovascular treatment strategies for supraaortic arterial occlusive disease. J Cardiovasc Surg (Torino). 2005 Jun;46(3):193200.

[7] Donmez H, Mavili E, Kaya MG, Soylu SO, Toker B. Endovascular treatment of coronary steal. Cardiovasc Revasc Med. 2011 Jan-Feb;12(1):67.e1-3. Epub 2010 Oct 20.

[8] Textor SC, Lerman L, McKusick M. The uncertain value of renal artery interventions: Where are we now? JACC Cardiovasc Interv 2009;2:175-182

[9] Kalra PA, Guo H, Kausz AT, Gilbertson DT, Liu J, Chen SC, Ishani A, Collins AJ, Foley RN. Atherosclerotic renovascular disease in United States patients aged 67 years or older: risk factors, revascularization, and prognosis. Kidney Int. 2005 Jul;68(1):293301.

[10] ASTRAL Investigators, Wheatley K, Ives N, Gray R, Kalra PA, Moss JG, Baigent C, Carr S, Chalmers N, Eadington D, Hamilton G, Lipkin G, Nicholson A, Scoble J. Revascularization versus medical therapy for renal-artery stenosis. N Engl J Med. 2009;361:1953-1962.

[11] Cicuto KP, McLean GK, Oleaga JA, Freiman DB, Grossman RA, Ring EJ. Renal artery stenosis: anatomic classification for percutaneous transluminal angioplasty. AJR Am J Roentgenol. 1981 Sep;137(3):599-601.

[12] Lederman RJ, Mendelsohn FO, Santos R, Phillips HR, Stack RS, Crowley JJ.Primary renal artery stenting: characteristics and outcomes after 363 procedures. Am Heart J. 2001 Aug;142(2):314-23.

[13] Norgren L, Hiatt WR, Dormandy JA, Nehler MR, Harris KA, Fowkes FG; TASC II Working Group. Inter-Society Consensus for the Management of Peripheral Arterial Disease (TASCII). J Vasc Surg. 2007 Jan;45 Suppl S:S5-67.

[14] Jaff M, Dake M, Pompa J, Ansel G, Yoder T. Standardized evaluation and reporting of stent fractures in clinical trials of noncoronary devices. Catheter Cardiovasc Interv. 2007 Sep;70(3):460-2.

[15] Mewissen MW. Primary nitinol stenting for femoropopliteal disease. J Endovasc Ther. 2009 Apr;16(2 Suppl 2):II63-81.

[16] Laird JR. Limitations of percutaneous transluminal angioplasty and stenting for the treatment of disease of the superficial femoral and popliteal arteries. J Endovasc Ther. 2006 Feb;13 Suppl 2:II30-40.

[17] Day CP, Orme R. Popliteal artery branching patterns -- an angiographic study. Clin Radiol. 2006 Aug;61(8):696-9. 
[18] Bosiers M, Deloose K, Verbist J, Peeters P. Update management below knee intervention. Minerva Cardioangiol. 2009 Feb;57(1):117-29. 


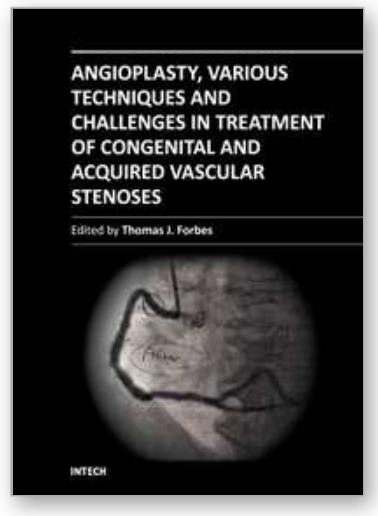

\section{Angioplasty, Various Techniques and Challenges in Treatment of Congenital and Acquired Vascular Stenoses}

Edited by Dr. Thomas Forbes

ISBN 978-953-51-0084-3

Hard cover, 236 pages

Publisher InTech

Published online 14, March, 2012

Published in print edition March, 2012

The field of performing transcatheter interventions to treat vascular lesions has exploded over the past 20 years. Not only has the technology changed, especially in the arena of balloon/stent devices, but the techniques of approaching complex lesions has evolved over the past decade. Lesions that no one would have imagined treating back in the 1990's are now being done routinely in the catheterization suite. This book provides an update on the current techniques and devices used to treat a wide variety of lesions. Though, at first, the outward appearance of the topics appears to be varied, they are all related by the common thread of treating vascular lesions. We hope, by publishing this book, to accomplish two things: First, to offer insight from experts in their field to treat, both medically and procedurally, complex vascular lesions that we frequently encounter. Secondly, we hope to promote increased communication between areas of medicine that frequently don't communicate, between adult interventional cardiologists, pediatric interventional cardiologists, interventional radiologists, and neurosurgeons. Much can be learned from our respective colleagues in these areas which can further our own world of interventions.

\section{How to reference}

In order to correctly reference this scholarly work, feel free to copy and paste the following:

Yoshiaki Yokoi (2012). Angiography for Peripheral Vascular Intervention, Angioplasty, Various Techniques and Challenges in Treatment of Congenital and Acquired Vascular Stenoses, Dr. Thomas Forbes (Ed.), ISBN: 978953-51-0084-3, InTech, Available from: http://www.intechopen.com/books/angioplasty-various-techniquesand-challenges-in-treatment-of-congenital-and-acquired-vascular-stenoses/angiography-for-peripheralvascular-intervention

\section{INTECH}

open science | open minds

\section{InTech Europe}

University Campus STeP Ri

Slavka Krautzeka 83/A

51000 Rijeka, Croatia

Phone: +385 (51) 770447

Fax: +385 (51) 686166

www.intechopen.com

\section{InTech China}

Unit 405, Office Block, Hotel Equatorial Shanghai

No.65, Yan An Road (West), Shanghai, 200040, China 中国上海市延安西路65号上海国际贵都大饭店办公楼 405 单元

Phone: +86-21-62489820

Fax: $+86-21-62489821$ 
(C) 2012 The Author(s). Licensee IntechOpen. This is an open access article distributed under the terms of the Creative Commons Attribution 3.0 License, which permits unrestricted use, distribution, and reproduction in any medium, provided the original work is properly cited. 